\title{
First current density measurements in the ring current region using simultaneous multi-spacecraft CLUSTER-FGM data
}

\author{
C. Vallat ${ }^{1}$, I. Dandouras ${ }^{1}$, M. Dunlop ${ }^{2}$, A. Balogh ${ }^{3}$, E. Lucek $^{3}$, G. K. Parks ${ }^{4}$, M. Wilber ${ }^{4}$, E. C. Roelof ${ }^{5}$, G. Chanteur ${ }^{6}$, \\ and H. Rème ${ }^{1}$ \\ ${ }^{1}$ Centre d'Etude Spatiale des Rayonnements, Toulouse, France \\ ${ }^{2}$ Rutherford Appleton Laboratory, Didcot, UK \\ ${ }^{3}$ Blackett Laboratory, Imperial College, London, UK \\ ${ }^{4}$ Space Sciences Laboratory, University of California, Berkeley, CA, USA \\ ${ }^{5}$ Applied Physics Laboratory, Johns Hopkins University, Baltimore, Maryland, USA \\ ${ }^{6}$ Centre d'Etude des Environnements Terrestre et Planétaires, Vélizy, France
}

Received: 10 August 2004 - Revised: 5 April 2005 - Accepted: 8 April 2005 - Published: 28 July 2005

\begin{abstract}
The inner magnetosphere's current mapping is one of the key elements for current loop closure inside the entire magnetosphere. A method for directly computing the current is the multi-spacecraft curlometer technique, which is based on the application of Maxwell-Ampère's law. This requires the use of four-point magnetic field high resolution measurements. The FGM experiment on board the four Cluster spacecraft allows, for the first time, an instantaneous calculation of the magnetic field gradients and thus a measurement of the local current density. This technique requires, however, a careful study concerning all the factors that can affect the accuracy of the $J$ estimate, such as the tetrahedral geometry of the four spacecraft, or the size and orientation of the current structure sampled. The first part of this paper is thus providing a detailed analysis of the method accuracy, and points out the limitations of this technique in the region of interest. The second part is an analysis of the ring current region, which reveals, for the first time, the large latitudinal extent of the ring current, for all magnetic activity levels, as well as the latitudinal evolution of the perpendicular (and parallel) components of the current along the diffuse auroral zone. Our analysis also points out the sharp transition between two distinct plasma regions, with the existence of high diamagnetic currents at the interface, as well as the filamentation of the current inside the inner plasma sheet. A statistical study over multiple perigee passes of Cluster (at about $4 \mathrm{R}_{E}$ from the Earth) reveals the azimuthal extent of the partial ring current. It also reveals that, at these distances and all along the evening sector, there isn't necessarily a strong dependence of the local current density value on the magnetic activity level. This is a direct consequence of the ring current morphology evolution, as well as the relative positioning of
\end{abstract}

Correspondence to: C. Vallat

(claire.vallat@cesr.fr) the spacecraft with respect to the bulk of the ring current. It also proves the existence of a substantial ring current at these distances, all over the evening and the post-midnight sector.

Keywords. Magnetospheric physics (Current systems; Energetic particles, trapped; Magnetospheric configuration and dynamics)

\section{Introduction}

The existence of a ring current forming around the Earth was first suggested by Singer (1957), who showed that a westward electric current was produced by the gradient drift of energetic particles $(\sim 1 \mathrm{keV}$ to a few hundred of $\mathrm{keV})$, trapped in the geomagnetic field. The equivalent current can be envisioned as toroidal-shaped, and flowing around the Earth at geocentric distances from about $2 \mathrm{R}_{E}$ to $9 \mathrm{R}_{E}$.

The global current system of the inner magnetosphere is, however, of a more complex nature, but can be described as a current system mainly driven by pressure gradients. During geomagnetic storm main phases, the ring current pressure is expected to be centred around midnight, at geocentric distances of the order of $\sim 3 \mathrm{R}_{E}$ (Lui et al., 1987). The resulting pressure gradient corresponds to a net westward current flowing on the outer edge of the ring current. At smaller geocentric distances, however, of the order of $\sim 2 \mathrm{R}_{E}$, an eastward current is formed, as the result of the reversed orientation of the pressure gradient vector. Off the equator, a current issued from the ring current connects to the ionosphere, forming a large part of the Region 2 current system (Iijima and Potemra, 1976). The ring current evolution is dependent on particle injections during geomagnetic activity increases and loss mechanisms (Daglis et al., 1999). In the inner magnetosphere, a co-existence of perpendicular currents (around 
the equator) and field-aligned currents (at higher latitudes) is thus expected, but its mapping, as well as its evolution as a function of the magnetic activity level, remain unclear. Prior to Cluster it was impossible to obtain a precise idea of the current response to magnetospheric changes, since simultaneous magnetic field measurements at multiple, geometrically favourable positions were generally unavailable.

The most common way to estimate the perpendicular current component inside the magnetosphere, using multispacecraft data, is the pressure gradient measurement. From the MHD momentum equation, and ignoring gravity and collisions, we can state that the perpendicular component of the current density at a boundary is :

$\boldsymbol{J}_{\perp}=\frac{\boldsymbol{B} \times \nabla \cdot \stackrel{\leftrightarrow}{P}}{B^{2}}+\rho_{m} \frac{\boldsymbol{B}}{B^{2}} \times \frac{d \boldsymbol{U}_{\perp}}{d t}$,

with $\frac{d U_{\perp}}{d t}$ representing the perpendicular component of the fluid acceleration with respect to the magnetic field $\boldsymbol{B}$, and $\overleftrightarrow{P}$ the pressure tensor. The first term on the right side represents the diamagnetic current component, and the second term represents the contribution to the current resulting from the coupling of the acceleration of the perpendicular fluid flow to the magnetic field. Thus, under static conditions, this expression can be reduced to:

$\boldsymbol{J}_{\perp} \approx \frac{\boldsymbol{B} \times \nabla \cdot \stackrel{\leftrightarrow}{P}}{B^{2}} \approx \frac{\boldsymbol{B}}{B^{2}} \times\left[\nabla_{\perp} \cdot P_{\perp}+\left(P_{/ /}-P_{\perp}\right) \cdot \frac{(\boldsymbol{B} . \nabla) \boldsymbol{B}}{B^{2}},\right]$

where $P_{/ /}$and $P_{\perp}$ are the parallel and perpendicular pressure, respectively. Experimentally, magnetic field data, as well as particle data, are required to perform this calculation.

This work has been performed previously by Lui et al. (1987). Using AMPTE/CCE data, Lui et al. (1987) estimated the perpendicular pressure (and $J_{\perp}$ ) from distribution functions of particles during two geomagnetic storms. Nevertheless, the limited energy range of the instrument $(25 \mathrm{keV}-1 \mathrm{MeV})$, as well as the working assumptions used in the calculations (such as stationarity between two successive points of measurement), did not allow them to obtain an instantaneous picture of the ring current. To reduce the limitations due to temporal effects, a simultaneous multispacecraft analysis is thus necessary.

Pressure gradients can, in principle, be computed almost for any instant using Cluster four spacecraft particle data. Nevertheless, this is not an easy task since it requires a very precise cross calibration of the particle instruments on the different spacecraft, so as to deduce correctly the plasma pressure gradient. Moreover, it is necessary to evaluate how plasma structures are moving after subtracting the spacecraft motion. Experimentally, this is done by determining the structure's motion with respect to the spacecraft. This implies that all spacecraft need a separation large enough to allow for a sufficient time-drift to measure the structure's velocity $\left(\Delta \mathrm{t}>\mathrm{t}_{\text {spin }}\right)$, but not so large as to violate the assumption of stationarity. As a consequence, this method is not accurate during high activity periods. We should also note that the parallel component of the current cannot be calculated by a pressure gradient estimate.

The other method developed from four-point measurements is based on magnetic field data and has been coined the curlometer technique (Robert and Roux, 1993; Robert et al., 1998b; Dunlop et al., 2002; Dunlop and Balogh, 1993). It is based on Maxwell-Ampère's law

$\mu_{0} \boldsymbol{J}=\nabla \times \boldsymbol{B}-\varepsilon_{0} \mu_{0} \frac{\partial \boldsymbol{E}}{\partial t}$

and it assumes stationarity in the region of interest ( $\partial \boldsymbol{E} \partial t=0$, i.e. assuming the field does not vary on time scales of the spacecraft motion). Moreover, this method assumes that all measurement points are situated inside the same current sheet. Thus, we can estimate the average current density through the tetrahedron formed by the four-point measurement configuration.

This method has been recently applied by Le et al. (2004), using magnetic field data from three different spacecraft surveying the region of interest at different times. This study permitted a global 3-D mapping of the ring current region over a wide $D_{s t} *$ range $\left(D_{s t} *\right.$ is the corrected $D_{s t}$ index from which the contribution of magnetopause current is removed).

Jorgensen et al. (2004) also established a ring current density mapping in the inner magnetosphere, but based on the $D_{s t}$ index and using statistical magnetic field data issued from the Combined Release and Radiation Effects Satellite (CRRES).

Nevertheless, the data used to calculate $\nabla \times \boldsymbol{B}$ at each point, in these two studies, were not acquired simultaneously, and since the $D_{s t} * / D_{s t}$ value was the only parameter used to classify the events, the mixing of magnetic field measurements corresponding to different storm phases, for a given current density calculation, cannot be excluded.

The use of simultaneous multi-spacecraft data allows one, for the first time, to reduce considerably the limitations induced by the current density estimate methods used so far.

The four spacecraft of the Cluster mission are all situated in a high-eccentricity polar orbit, and their configuration can be represented by the spacecraft separation vectors, allowing for a relative positioning. From Cluster magnetic field data, the curlometer technique is applied using data obtained simultaneously on board the four spacecraft. Nevertheless, the measurement accuracy of the current density using the curlometer technique can be substantially affected by different sources: the tetrahedral geometry of the four spacecraft, the size (in time and space) of the current structure sampled, the linear interpolation made between various measurement points, and the eventual experimental errors inherent to the magnetometer. To validate the technique a detailed analysis of these error sources has to be done in the ring current region, as well as their influence on the current density value, while the Cluster spacecraft were passing at perigee. This work is presented in Sect. 4. After the validation of the technique, we make an analysis of the ring current evolution for two events corresponding to different magnetospheric activity levels. Our analysis confirms the existence of a partial 
ring current in the evening and post-midnight sector, and reveals the current driven by drifting particles as being the main part of the total current, even for quiet events. A latitudinal analysis of the current over the entire diffuse auroral zone has also been made, focusing on plasma boundaries and diamagnetic signatures. Very narrow structures, such as current filamentations, are revealed at the same time on current signatures and on particle data. Finally, a statistical study, over multiple perigee passes of Cluster, allows for a global 3-D mapping of the current in the inner magnetosphere for a large magnetic local time sector.

\section{Cluster orbit and instrumentation}

The Cluster mission is based on four identical spacecraft launched on similar elliptical polar orbits with a perigee at about $4 \mathrm{R}_{E}$ and an apogee at $19.6 \mathrm{R}_{E}$ (Escoubet et al., 2001). This allows Cluster to cross the ring current region from south to north during every perigee pass, and to obtain its latitudinal profile. Moreover, due to the orbit precession of the spacecraft over the year, all magnetic local times can be studied. As a consequence, an overview of the ring current region at $r \sim 4 \mathrm{R}_{E}$, over all MLT and latitudinal sectors, is feasible.

The inter-spacecraft separation strategy has been planned in order to allow for the study of the various plasma structures encountered by Cluster along the orbit. The maneuvers to change the inter-spacecraft separation take place once or twice a year, depending on the spatial scales of the plasma structures to be studied. The tetrahedron formed by the four spacecraft can thus have characteristic sizes ranging between $100 \mathrm{~km}$ and a few $\mathrm{R}_{E}$. As a consequence, only for limited time periods does the mission allow for an analysis of the ring current. In the time period from February to June 2002 the Cluster inter-spacecraft separation was reduced so as to obtain a regular tetrahedron of a $100-\mathrm{km}$ characteristic size when traversing the cusp. This configuration is the only one small enough so as to permit all spacecraft to be situated inside the same current sheet, as will be shown in Sect. 4.1. Nonetheless, this assumption needs to be verified case by case. The resulting tetrahedron formed by the cluster is elongated, with a $\sim 70 \mathrm{~km}$ width and a $\sim 240 \mathrm{~km}$ height when crossing perigee, mainly along the GSE z-axis. Time periods of interest for the ring current study will be from February to June 2002 (see Sect. 4).

On board each spacecraft, eleven experiments permit a wide variety of measurements of the plasma parameters (particles and fields). Among them, a fluxgate magnetometer (FGM), as well as two ion spectrometers (HIA and CODIF), are present.

\subsection{FGM}

The FGM experiment on board Cluster consists of two triaxial fluxgate magnetometers and an onboard data-processing unit on each spacecraft. High vector sample rates (up to 67 vectors. $\mathrm{s}^{-1}$ ) at high resolution (up to $8 \mathrm{pT}$ ) allow for a precise measurement of the ambient magnetic field, with background interference from the spacecraft minimised by placement of the magnetometers on a five meter boom, avoiding interference with the spacecraft. The magnetic field component along the spin axis (which is almost perpendicular to the ecliptic) will carry the main part of the error made on the measurement, because offsets in the spin plane measurements are easily removed by noting spin-period oscillations. In addition to the on-ground calibrations made to determine the expected maximal offset on each spacecraft (up to $0.1 \mathrm{nT}$ ), in-flight calibrations of the magnetometers are regularly applied in order to maximise the accuracy of the magnetic filed measurements. A detailed description of the instruments and its performances is given by Balogh et al. (1997, 2001).

\subsection{CIS}

The Cluster Ion Spectrometer experiment consists of two complementary ion sensors, the COmposition and DIstribution Function analyzer (CODIF) and the Hot Ion Analyzer (HIA). CODIF gives a mass per charge composition with a $22.5^{\circ}$ angular resolution, and HIA offers a better angular resolution $\left(\sim 5^{\circ}\right)$ but without mass discrimination. CIS is capable of measuring the full three-dimensional ion distribution of the major ion species, from thermal energies $(\sim 1 \mathrm{eV})$ to about $40 \mathrm{keV}$, with one spacecraft spin (4s) time resolution (Rème et al., 2001).

\section{Method description: the curlometer technique}

The curlometer technique has been described in detail by Dunlop et al., 1988; 2002 and by Robert et al., 1998a. Here we only briefly outline the method.

Maxwell-Ampère's law states that, assuming stationarity, Eq. (3):

$\mu_{0} \boldsymbol{J}=\nabla \times \boldsymbol{B}$,

where $\boldsymbol{J}$ represents the current density and $\boldsymbol{B}$ the magnetic field. Thus, in a discrete Cartesian co-ordinate system, we state that:

$(\operatorname{curl} B) x \approx \frac{\Delta B z}{\Delta y}-\frac{\Delta B y}{\Delta z}$
$(\operatorname{curl} B) y \approx \frac{\Delta B x}{\Delta z}-\frac{\Delta B z}{\Delta x}$
$(\operatorname{curl} B) z \approx \frac{\Delta B y}{\Delta x}-\frac{\Delta B x}{\Delta y}$.

These equations can be applied to the Cluster data (four simultaneous points of the magnetic field measurements) to evaluate the magnetic field gradients (over the spacecraft) and thus the current density through the tetrahedron formed by the four spacecraft.

To avoid any coordinate system dependency, and using Stokes' theorem, we state that:

$\mu_{0} \iint \boldsymbol{J} \cdot d s=\oint \boldsymbol{B} \cdot d l$ 
which can be approximated by :

$\mu_{0} \boldsymbol{J}_{\text {average. }}\left(\Delta \boldsymbol{r}_{i} \times \Delta \boldsymbol{r}_{j}\right)=\Delta \boldsymbol{B}_{i} . \Delta \boldsymbol{r}_{j}-\Delta \boldsymbol{B}_{j} . \Delta \boldsymbol{r}_{i}$,

where $\boldsymbol{J}_{\text {average }}$ represents the measured mean current over

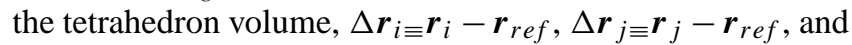
similarly $\Delta \boldsymbol{B}_{j \equiv} \boldsymbol{B}_{j}-\boldsymbol{B}_{r e f}$.

Using Eq. (6), we can also estimate $\nabla \cdot \boldsymbol{B}$ by:

$$
\begin{array}{r}
\langle\nabla \cdot \boldsymbol{B}\rangle_{a v}\left|\Delta \boldsymbol{r}_{i} \cdot\left(\Delta \boldsymbol{r}_{j} \times \Delta \boldsymbol{r}_{k}\right)\right|= \\
\left|\sum_{\text {cyclic }} \Delta \boldsymbol{B}_{i} \cdot\left(\Delta \boldsymbol{r}_{j} \times \Delta \boldsymbol{r}_{k}\right)\right|,
\end{array}
$$

where $\langle\nabla \cdot \boldsymbol{B}\rangle_{a v}$ is the differential estimate of $\nabla \cdot \boldsymbol{B}$ for the tetrahedron (Dunlop et al., 1988, 2002). Magnetic field and relative positions between spacecraft are computed relatively to one spacecraft of reference, usually the spacecraft number one. The influence of the reference spacecraft selection on the current density determination will be discussed in Sect.4.2.

\section{Accuracy limitations of the method}

The principal assumption required for the use of the curlometer technique is a linear variation of the magnetic field inside the tetrahedron, which implies that the current density is constant inside the tetrahedron. This is equivalent to stating that the four spacecraft are passing simultaneously through the same current sheet. This condition is well satisfied as far as the inter-spacecraft separation is short enough compared to the plasma structures. A qualitative way to check this assumption is to examine the particle data for each event. But, because $\boldsymbol{B}$ is solenoidal, this can be checked more rigorously by computing the field's divergence. In fact, non-zero values of $\operatorname{div}(\boldsymbol{B})$ would characterize truncation errors successive to the non-linearity of the field inside the tetrahedron. Nevertheless, this quantity, while an approximate indicator of the current density truncation error, is not proportional to it.

\subsection{Tetrahedron size and shape}

During some part of the orbit, extreme distortions of the tetrahedron can occur (due to the spacecraft configuration and its orientation relative to the magnetic field structure), resulting in a poorer accuracy on the determination of one, or more, components of $\boldsymbol{J}$. Since the $\operatorname{div}(\boldsymbol{B}) \equiv 0$ computation is not the only condition required for a good estimate of the current density, every perigee pass will need a careful analysis of the shape and orientation of the tetrahedron relative to the magnetic environment, in order to determine how each component is affected by the tetrahedron distortions. In addition to the size scale of the tetrahedron, two parameters were introduced to characterise the tetrahedron geometry, the elongation and the planarity. The relationship between these two parameters and the current density error estimate have been discussed in detail by Robert et al. (1998a; 1998b) and by Chanteur (2000).

\subsubsection{Size}

The size of the tetrahedron has to be small enough so as to permit the gradients (inside the tetrahedron) to be as linear as possible. This means that the size of the tetrahedron must be small enough so as to let the four spacecraft to be situated simultaneously in the same current sheet. To verify this condition, we can check the position of each spacecraft with respect to the plasma boundaries, by using the CIS/CODIF and RAPID data. Since CIS is not operational on SC2, we used, on board this spacecraft, the lowest energy range of the RAPID instrument (Wilken et al., 1997, 2001) to check the position of the boundary. As a first approach, we can assume that the four spacecraft are situated in the same current sheet if they are situated inside the same plasma region.

However, the smaller the tetrahedron size, the larger the absolute error made on the $\Delta \boldsymbol{B}$ and the $\Delta \boldsymbol{r}$ estimation and on the $\boldsymbol{J}_{\text {real }}-\nabla \times \boldsymbol{B}_{\text {exp }}$ quantity, which should be minimised ( $B_{\text {exp }}$ representing the measured magnetic field). Thus, there is an optimal inter-spacecraft separation value for which the error made on the relative measurements is satisfactory and the gradients are almost linear. In the ring current region, and considering the tetrahedron deformation during the perigee pass, the 100-km separation strategy (estimated spacecraft distance while crossing the cusp, i.e. at about $10 \mathrm{R}_{E}$ ), as well as the 200-km strategy (estimated spacecraft distance while crossing the tail, i.e. at about $18 \mathrm{R}_{E}$ ), will be the only ones allowing for the spacecraft to be situated inside the same current sheet. Above these distances, the gradients inside the tetrahedron are no longer linear.

\subsubsection{Configuration}

As the tetrahedron shape is evolving along the inbound leg of the Cluster orbit and approaches perigee, it is subject to an elongation (larger acceleration of the leading spacecraft) followed by a flattening (deceleration of the leading spacecraft just after perigee). The tetrahedron thus cannot be considered as regular, especially when crossing perigee. Figure 1 is adapted from the study made by Robert et al. (1998b). The upper panels indicate the values of the elongation and planarity parameters for the 18 March 2002 perigee pass (100$\mathrm{km}$ separation strategy). The bottom panel gives an estimation of the error made on the $\frac{\Delta J}{J}$ ratio as a function of the elongation and the planarity factors, considering an homogenous current distribution, and for $\mathrm{J} \sim 10^{-8} \mathrm{~A} / \mathrm{m}^{2}$.

By examining their influence on the current density estimate, it appears that, for a $100-\mathrm{km}$ separation strategy (18 March 2002 tetrahedron configuration), the maximum error made on the current during a perigee pass can go up to $20 \%$. Nevertheless, for the $200-\mathrm{km}$ separation strategy period, this relative error reaches $60 \%$. This non-linear increase of the relative error is mainly due to the fact that the inter-spacecraft separation distances are adopted during different parts of the orbit for the 100 - and $200-\mathrm{km}$ separation strategy periods. Since the $200-\mathrm{km}$ separation is achieved during the tail crossing (i.e. at about $18 \mathrm{R}_{E}$ ), it yields to a much higher elongation 

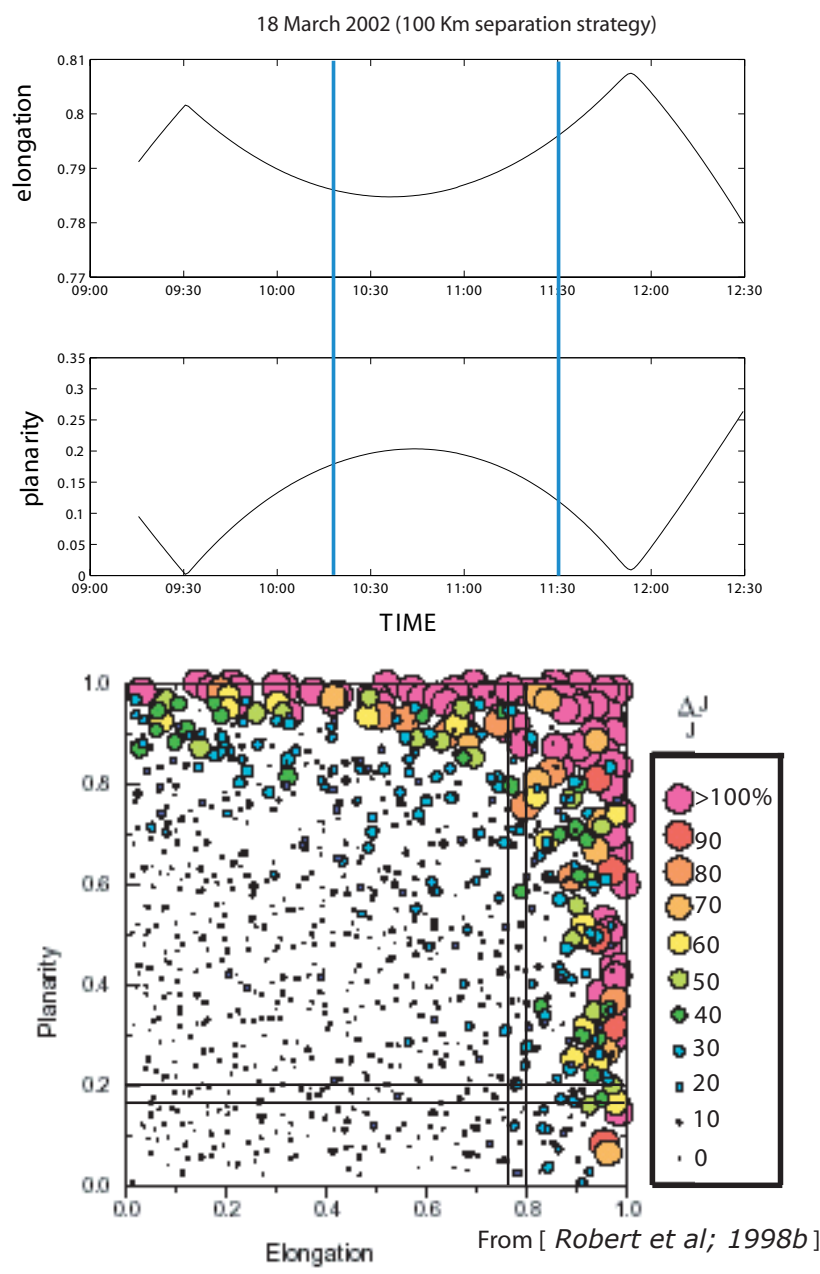

Fig. 1. Cluster spacecraft tetrahedron parameters for the 18 March 2002 perigee pass (upper panels), during which the spacecraft separation strategy was $100 \mathrm{~km}$. The two blue vertical lines delimit the ring current traversal by the spacecraft. Bottom panel shows results of the study made by Robert et al. (1998b), which evaluated the influence of the tetrahedron shape (characterised by the tetrahedron parameters) on the estimate of $|J|$. Black lines demarcate the extreme values taken by the two parameters for spacecraft separation distances during the 18 March 2002 ring current interval. This separation can go up to $250 \mathrm{~km}$ at perigee, due to the keplerian evolution of the orbit (acceleration of the leading spacecraft). We can notice that the maximum uncertainty on $|J|$ is never more than $20 \%$.

of the tetrahedron at perigee than the $100-\mathrm{km}$ one (which is made during the cusp crossing), and thus leads to a much larger relative error on the $J$ estimate. As a consequence, the only separation allowing a current density estimate accurate enough is the 100-km one (February to June 2002).

Moreover, considering that the tetrahedron is elongated along the $\mathrm{Z}$-axis while crossing perigee, the main part of the error made on the $J$ estimate will be carried by the $J_{z}$ component, determined by the $\mathrm{x}$ and $\mathrm{y}$ gradients (see Eq. (5)). Also, since we will focus on the $J$ component perpendicular to $\boldsymbol{B}$ (i.e. $J_{x}$ and $J_{y}$ near perigee), the accuracy will be considered as satisfactory for the two other components of the current estimate (see Fig. 1).
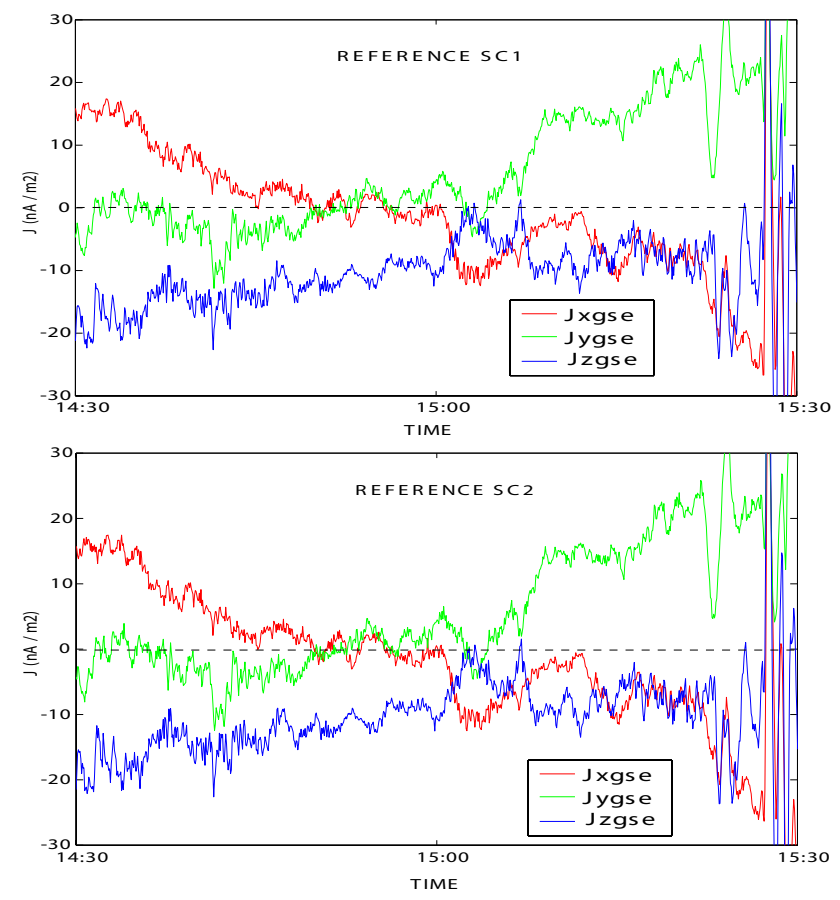

Fig. 2. Current density (in the GSE coordinate system) for the 13 April 2002 perigee pass, as computed using two different spacecraft of reference: SC1 (upper panel) and SC2 (lower panel).

\subsection{Error induced by the reference spacecraft selection}

As mentioned earlier, the curlometer technique requires the selection of a reference spacecraft (see Eq. (7)), in order to apply the Stokes theorem using Maxwell's equation. This implies that the selected spacecraft will have a mean weight in the calculation that is more important with respect to the three other spacecraft. Considering the tetrahedron distortion, one would expect that the estimated current density might have different values depending on the selected reference spacecraft. A comparison of the perigee current density values, as computed using two different spacecraft of reference for the same event, is presented in Fig. 2. It appears that changing the reference spacecraft has an insignificant influence on the current calculation.

\subsection{Dipole field truncation errors contribution}

By definition, for a dipolar magnetic field, and outside the current source of the dipole:

$\nabla \times \boldsymbol{B}_{\text {dip }}=0$.

As a consequence, there shouldn't be any contribution from the dipole to the current locally measured by Cluster. Non zero values of this contribution to $\boldsymbol{J}$ would be representative of truncation errors induced by the curlometer method. To check that the truncation errors for the dipole are not relevant, we subtracted the dipolar field contribution from the total magnetic field and compared the current deduced from this field with the one computed from the FGM data (see 


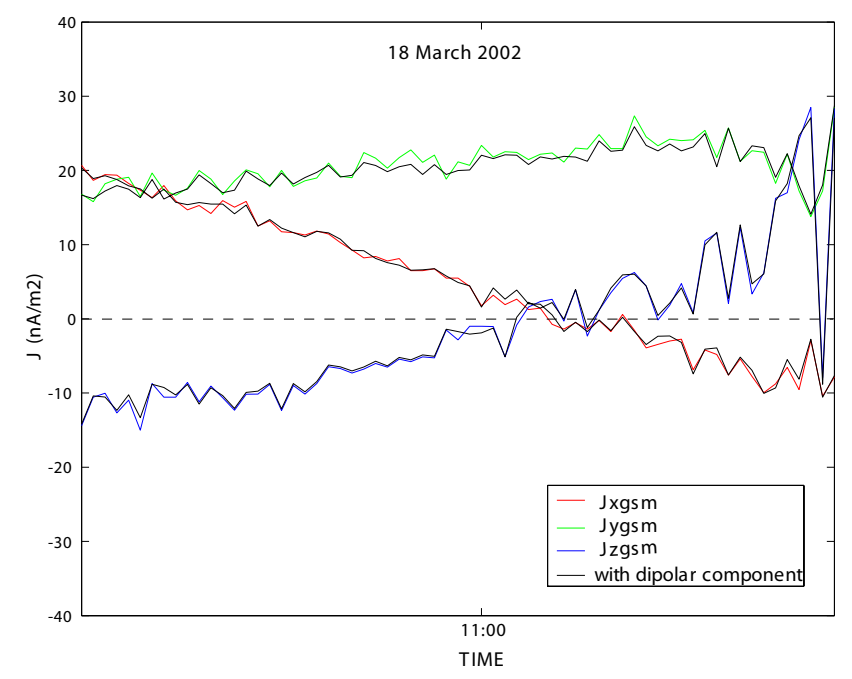

Fig. 3. Current density (in the the GSM coordinate system) as computed using two different magnetic field data inputs: FGM data (black curves) and FGM data from which we subtracted the dipolar field contribution (colored curves).

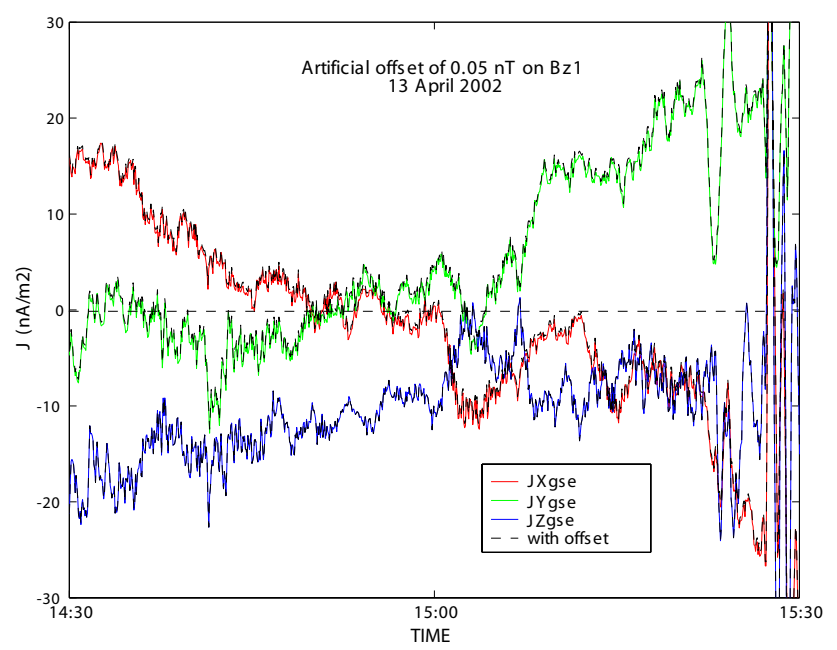

Fig. 4. Current density (in the GSE coordinate system) as computed using two different magnetic field data inputs: FGM data (colored curves) and FGM data to which we added an artificial offset of $0.05 \mathrm{nT}$ on the $B_{z}$ component (on SC1, black dashed curves).

Fig. 3). The results demonstrate that the truncation errors do not affect significantly the current density calculation, and confirm the consistency of the calculations.

\subsection{Error made on the magnetic field determination}

Apart from the truncation errors resulting directly from the tetrahedron geometry and the application of the (linear) method, experimental errors on the magnetic field measurements could affect the current density estimation.

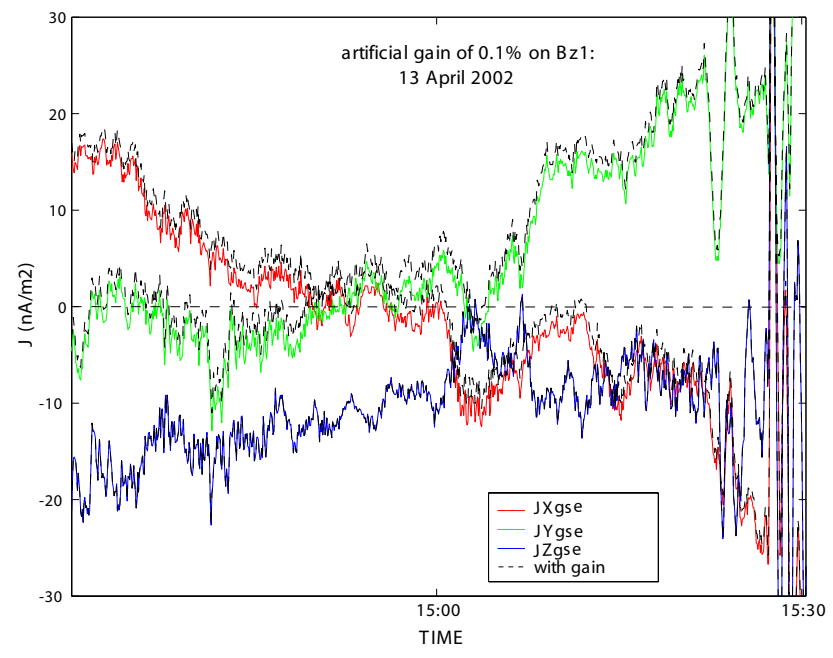

Fig. 5. Current density (in the GSE coordinate system) as computed using two different magnetic field data inputs: FGM data (colored curves) and FGM data to which we added an artificial gain of $0.1 \%$ on the $B_{z}$ component (on SC1, black dashed curves).

\subsubsection{Magnetometer offset}

To study the influence of an offset in the magnetic field measurements on the current density estimate, we compared $\boldsymbol{J}$ calculated using the field as measured by FGM, and calculated using the experimental field, to which we added an artificial offset of $0.05 \mathrm{nT}$ (added only to one spacecraft's magnetic field data). We chose to add the artificial offset to the $B_{z}$ component, since this is the component which carries the largest uncertainty (component situated along the Cluster spin axis). The results are shown in Fig. 4. It appears that the general trend of the current density components is not affected in a significant way by the introduction of this artificial overestimated offset. Furthermore, the calibration tests on FGM evaluated the offset uncertainty to no more than $0.02 \mathrm{nT}$. As a consequence, we can consider that the current density values will not be affected by any magnetometer offset.

\subsubsection{Magnetometer gain}

The same kind of study has been made with an artificial overestimated gain (of $0.1 \%$ ) added to the data of one of the spacecraft. This has been done on each spacecraft, and the data presented in Fig. 5 show the results for a gain added on $B_{z}$ (spacecraft 1 ). It appears that a gain variation would not affect significantly the general trend of the current density profile, although it would slightly shift the absolute values. Moreover, the gain uncertainty for FGM is evaluated to be no more than $0.02 \%$.

We have thus demonstrated that the main error sources coming from the magnetometer accuracy are not influencing the current density estimates in a significant way. 


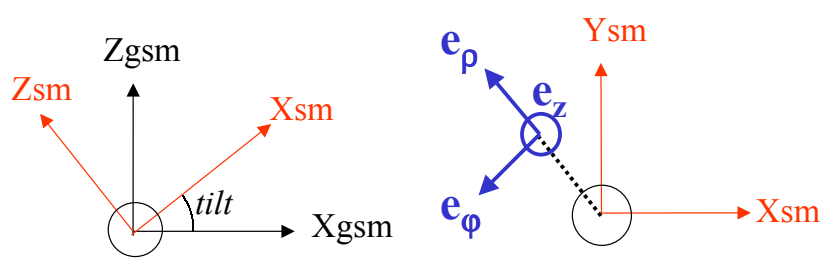

Fig. 6. Coordinate transformations from Geocentric Solar Magnetospheric into Solar Magnetic, and from Solar Magnetic into the local cylindrical coordinate system $\left[\boldsymbol{e}_{\rho}, \boldsymbol{e}_{\varphi}, \boldsymbol{e}_{z}\right]$, used in our analysis. The black circle represents the Earth, and the blue circle the Cluster spacecraft tetrahedron.

\section{Observations and analysis of the curlometer data for two events}

For our analysis, we selected two events, both corresponding to Cluster perigee passes in the evening sector, but with very different magnetic activity levels.

\subsection{Co-ordinate transformations}

Assuming that the ring current carried by particles drifting around the Earth is centred close to the magnetic equatorial plane and has a finite latitudinal extent above and below it, it is necessary to define a coordinate system which is well adapted to represent the ring current. As a consequence, a cylindrical system has been defined (see Fig. 6). Using solar magnetic coordinates of the current $\left(Z_{S M}\right.$ being defined as the dipole axis direction and $Y_{S M}$ being perpendicular to the plane containing the Earth-Sun line and the dipole axis, with a positive sense opposite to the Earth's orbital motion), we define the local cylindrical system as follows: $\boldsymbol{e}_{z}$ is parallel to the $Z_{S M}$ axis; $\boldsymbol{e}_{\rho}$ represents the radial component of the current on the plane parallel to the $\left(\mathrm{X}_{S M}, \mathrm{Y}_{S M}\right)$ plane, oriented anti-earthward; $\boldsymbol{e}_{\varphi}$ represents the component such that $\left(\boldsymbol{e}_{\rho}, \boldsymbol{e}_{\varphi}, Z_{S M}\right)$ is a direct trihedron, i.e. $\boldsymbol{e}_{\varphi}$ points eastward. This system $\left(\boldsymbol{e}_{\rho}, \boldsymbol{e}_{\varphi}, \boldsymbol{e}_{z}\right)$ has been represented schematically in Fig. 6.

\subsection{Quiet event: 18 March 2002}

\subsubsection{Context}

During the 18 March 2002 event the geomagnetic indices recorded a quiet period, with $K_{p}=1+$ and $D_{s t} \sim 10 \mathrm{nT}$. Nevertheless, a succession of small substorms occurred during this interval. For this period, the four Cluster spacecraft had a separation distance of about $100 \mathrm{~km}$ when crossing the cusp area. The spacecraft separation for this event was maximum during the perigee pass (as a result of the Keplerian evolution of the orbit), but did not exceed $260 \mathrm{~km}$. SC1 (spacecraft 1) was crossing the equator first, followed by SC4, SC2 and SC3, respectively. SC4 was crossing the equator at 10:48 UT, in the evening sector (MLT 23). Figure 7 presents CODIF, RAPID and FGM data from SC 4 for this event. From top to bottom, it shows, for the proton population, the energy-time

$$
\text { CIS-CODIF TANGO (SC 4) 18/Mar/2002 }
$$
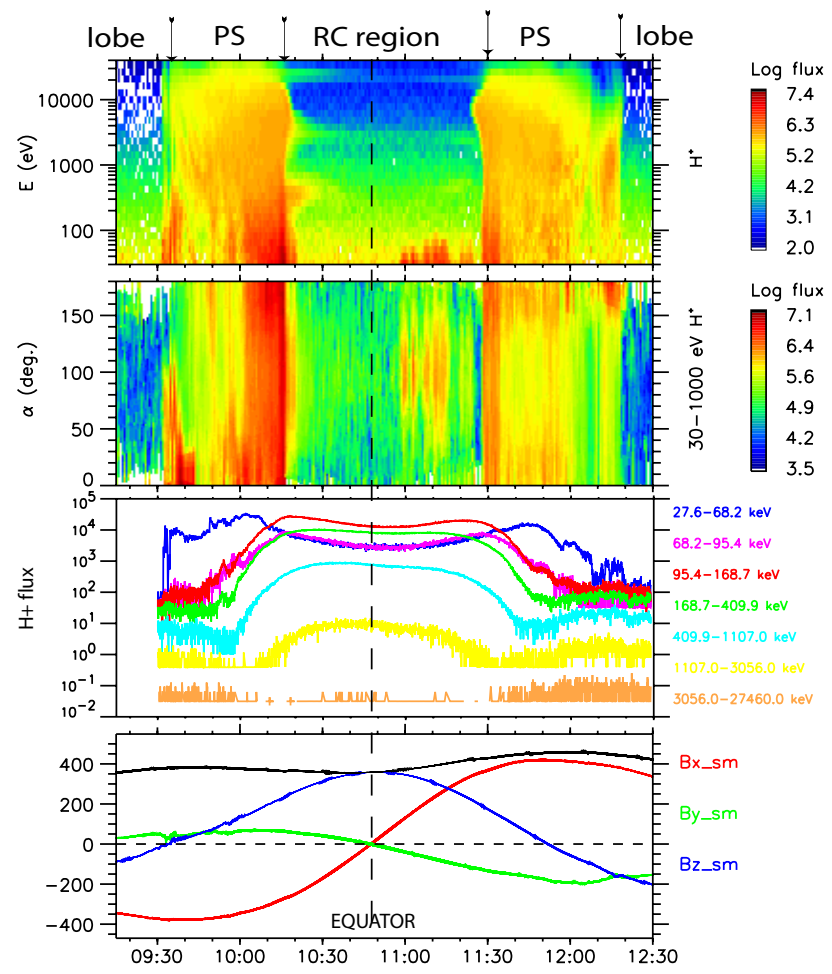

$\begin{array}{lrrrrr}\text { L } & 7.77 & 4.91 & 4.44 & 6.26 & 13.21 \\ \text { Ilat } & (-) 68.98 & (-) 63.18 & 61.67 & 66.44 & 74.03 \\ \text { Mlat } & -36.71 & -16.33 & 7.51 & 31.29 & 51.66 \\ \text { R } & 4.99 & 4.52 & 4.36 & 4.56 & 5.06\end{array}$

Fig. 7. Cluster SC4 data for the 18 March 2002 event: $\mathrm{H}^{+}$energy-

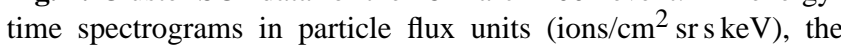
pitch-angle distribution (in particle flux units) for the 30 to $1000 \mathrm{eV}$ energy range, the $\mathrm{H}^{+}$energy-time spectrogram from the RAPID instrument (27.6-3056.0 keV) and the magnetic field components in the SM coordinate system. L-shell, invariant latitudes, magnetic latitudes and geocentric distances are indicated below. The dashed line represents the equatorial crossing.

spectrograms (from a few $\mathrm{eV}$ to $\sim 40 \mathrm{keV}$ ) and the pitchangle distribution (for the 30 to $1000 \mathrm{eV}$ energy range) in particle flux units (ions $/ \mathrm{cm}^{2} \mathrm{srs} \mathrm{keV}$ ) from the CIS-CODIF instrument, the energy-time spectrograms from the RAPID instrument (from $\sim 27.6 \mathrm{keV}$ to $\sim 3056 \mathrm{keV}$, in particle flux units); the magnetic field components, in SM coordinates (as measured by FGM), the L-shells, invariant latitudes, magnetic latitudes and geocentric distance values. Cluster was in the southern lobe until 09:31 UT, when it crossed a first boundary, situated at Ilat $\approx 68.6^{\circ}$ (Southern Hemisphere, hereafter referred as $\mathrm{SH}$ ), entering into the southern inner plasma sheet boundary layer. CODIF recorded then a succession of very thin plasma layers, especially at low energy ranges. These numerous alternations between field-aligned particles $\left(\alpha \approx 0^{\circ}\right)$ and perpendicular protons at low energies (up to about $1 \mathrm{keV}$ ), as shown in the pitch angle distribution plot, reveal the existence of two distinct populations. 


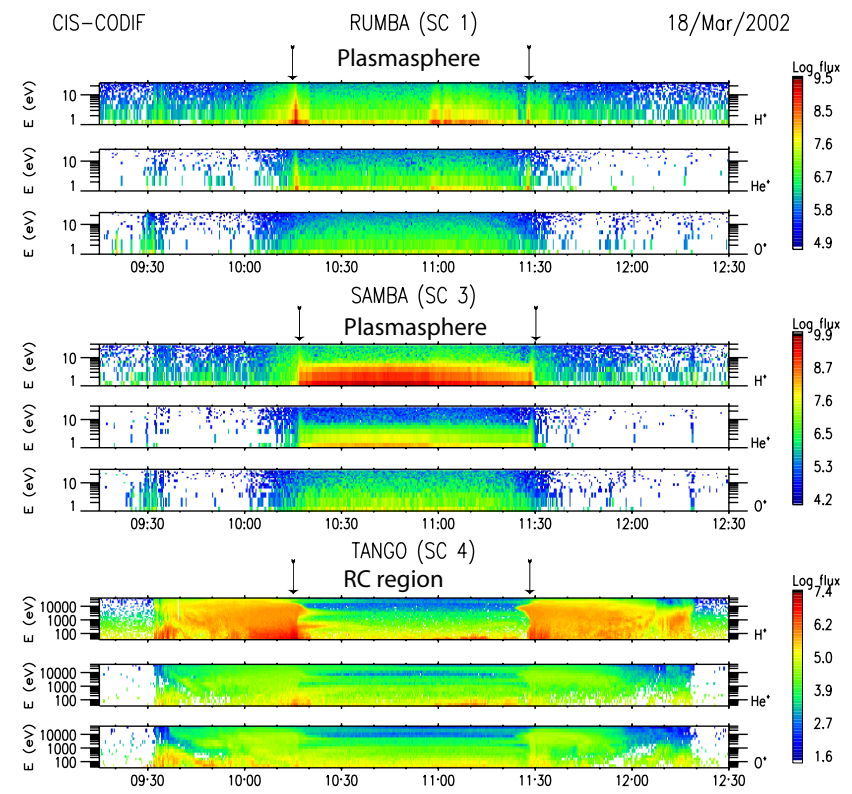

Fig. 8. CIS-CODIF data for the 18 March 2002 event: $\mathrm{H}^{+}, \mathrm{He}^{+}, \mathrm{O}^{+}$energy-time spectrograms in particle flux units (ions $/ \mathrm{cm}^{2}$ sr s keV) from SC1, SC3 and SC4. CODIF on SC1 and SC3 were using the RPA mode, allowing measurements of plasma particles from $\sim 1 \mathrm{eV}$ to $\sim 25 \mathrm{eV}$, characteristic of the plasmasphere. CODIF on SC4 was operating in a normal magnetospheric mode $(25 \mathrm{eV}-40 \mathrm{keV})$ which allows the detection of the ring current particles. We thus are using the $\mathrm{SC} 1$ and 3 to monitor the position of the plasmasphere/plasmapause, and SC4 to monitor the ring current. As it appears on these 3 spectrograms, and taking into account that the spacecraft separation was only a few hundred kilometers, the plasmasphere and the ring current boundaries were almost collocated for this event, at the MLT sector of the Cluster trajectory (see text for details).

The field-aligned particles are present at invariant latitudes as low as $64^{\circ}(\mathrm{SH})$, up-flowing from the ionosphere, but from Ilat $\approx 66^{\circ}$ (SH, 09:43 UT) to $62^{\circ}$, the dominant population is isotropic. At 10:16 UT a sharp boundary was encountered by SC 4, characterizing the transition between the inner plasma sheet and the ring current region. A flux gradient, up to 3 orders of magnitude for the highest CODIF energy ranges (above $4 \mathrm{keV}$ ), was observed, and CODIF recorded very low proton flux values all over the ring current traversal. Cluster stayed in the ring current region until 11:28 UT, where it crossed the northern part of the inner plasma sheet, revealing, like in the Southern Hemisphere, the existence of up-flowing particles from the ionosphere $\left(\alpha \approx 180^{\circ}\right)$ at low energies (30$1000 \mathrm{eV})$.

When Cluster was entering in the ring current region, the RAPID data revealed an important increase in the proton flux at higher energies (above $\sim 95 \mathrm{keV}$ ), whereas a sharp decrease was observed simultaneously by CODIF at lower energy ranges (up to $40 \mathrm{keV}$ ).

Figure 8 shows the ion spectrograms $\left(\mathrm{H}^{+}, \mathrm{He}^{+}\right.$and $\left.\mathrm{O}^{+}\right)$ for the three operating CODIF instruments. On both SC 1 and SC 3, CODIF was run in the RPA mode (energy range
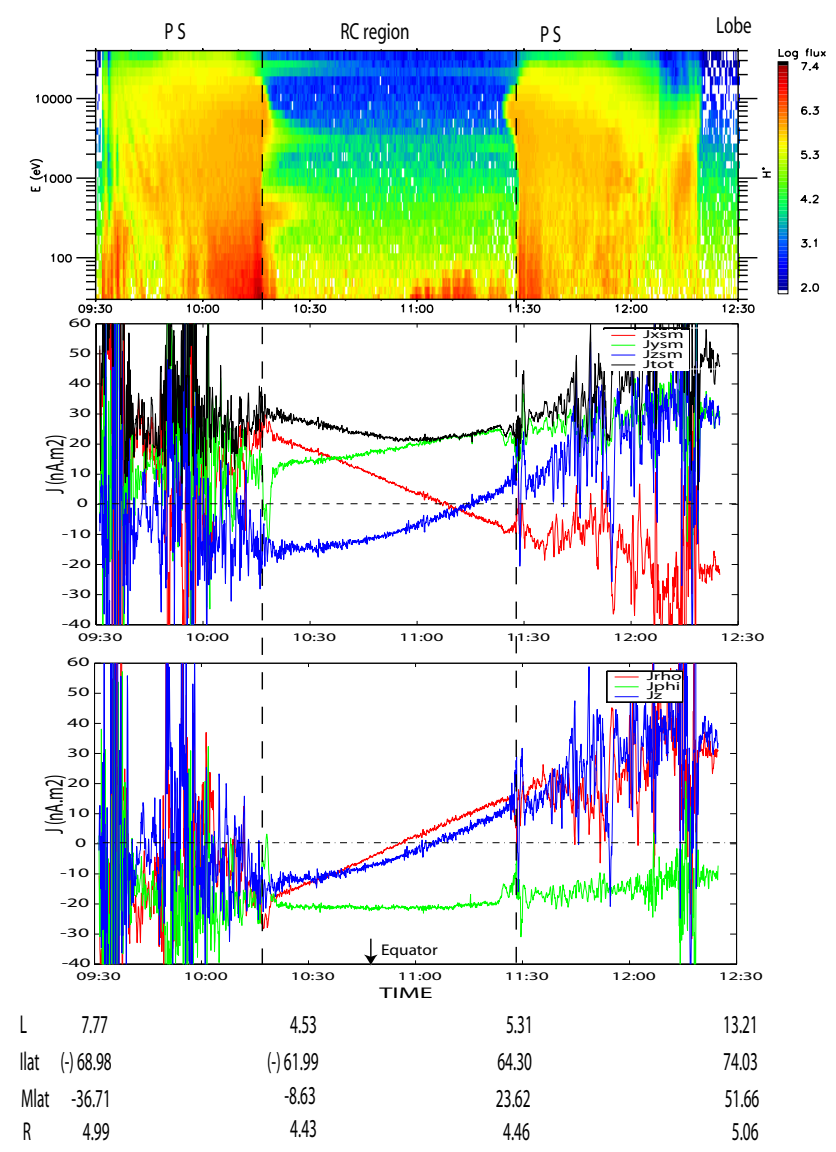

Fig. 9. Cluster data for the 18 March 2002 event: $\mathrm{H}^{+}$energy-time spectrogram for SC4 in particle flux units (ions $/ \mathrm{cm}^{2} \mathrm{srs} \mathrm{keV}$ ), current density components in the SM coordinate system and in $\mathrm{nA} / \mathrm{m}^{2}$ (second panel), and in the local cylindrical coordinate system (bottom panel). Black dashed lines demarcate the ring current region. L-shell, invariant latitudes, magnetic latitudes and geocentric distances are indicated below.

$\approx 1$ to $25 \mathrm{eV}$ ) to allow for a sampling of particles constituting the plasmaspheric population (Dandouras et al., 2005). SC3 is revealing a very sharp transition on the low energy population flux, corresponding to the plasmapause crossing, and observed for the three ion species. Comparing boundaries observed by CODIF on SC4 (using normal mode) and SC1 and 3 (using RPA mode), the plasmapause crossing (seen on SC3) corresponds locally to the transition between the plasma sheet and the ring current (observed on SC4).

Examining in detail the magnetic field data, we note some irregularities on each component. By comparing FGM data with an IGRF field, it appears that while passing through the equator plane, the measured magnetic field is weaker than the corresponding IGRF one. Note that even if the IGRF model does not take into account external sources of the magnetic field (and thus does not reflect the total field for distances beyond $4 \mathrm{R}_{E}$ ), it can be used to position the external sources with respect to the measurement point position. This result $\left(\left\|\mathrm{B}_{F G M}\right\|<\left\|\mathrm{B}_{I G R F}\right\|\right)$ can be interpreted as a consequence of the spacecraft relative position with respect to 
the bulk of the current which creates the induced magnetic field. Even if the induced magnetic field in the region of the spacecraft is quite complex, there are four main sources of it, (e.g. Liemohn et al., 2001; Milillo et al., 2003): (i) a westward current known as the ring current part (Roelof, 1989), whose flow tends to decrease the magnetic field in the region situated earthward to it; (ii) an eastward current situated earthward with respect to the westward component (and much weaker), which tends to increase the magnetic field amplitude earthward and decrease it tailward; (iii) a current flowing in the tail, whose influence on the inner magnetosphere is to decrease the magnetic field amplitude (Lui, 1984; Mitchell et al., 1990); and (iv) the field-aligned currents (Iijima and Potemra; 1976). Note that the influence of the tail current is not as important as that of the ring current, since this current is flowing farther from the inner magnetosphere. For this event, the total magnetic field, as measured by FGM, is weaker than the corresponding IGRF one while passing through the equatorial plane. This allows us to conclude that the spacecraft is passing earthward with respect to the bulk of the westward ring current. Its position with respect to the eastward flowing ring current cannot be directly deduced from the magnetic field data, and we need, therefore, to compute the current flowing across the tetrahedron by using the curlometer.

In order to check the applicability of the curlometer for this event, we estimated the $\operatorname{div}(\boldsymbol{B}) / \operatorname{curl}(\boldsymbol{B})$ values, using Eqs. (7) and (8). This quantity is very low, even if non-zero. Thus, we can conclude that the use of the curlometer technique under these conditions is possible. To verify this with ion energy measurements, we used CODIF data from SC4 (the only spacecraft on which CODIF, during this event, was operating in a full-energy mode) and HIA and/or RAPID data from $\mathrm{SC} 1, \mathrm{SC} 2$ and $\mathrm{SC} 3$. Note that the CIS experiment is not operational on SC2. In spite of a partial saturation of HIA due to the presence of penetrating particles from the radiation belts, we were able to verify the existence, in the SC1 and SC3 data, of the same boundaries detected by CODIF on SC4 (SC1 and SC3 HIA data not shown). We also detected simultaneously a boundary at higher energy ranges, using RAPID data on the four spacecraft. Assuming that in this region current sheets separate different particle layers, we can confirm that all spacecraft were situated in the same current sheet while crossing perigee. As a consequence, simultaneous Cluster measurements can be applied to determine a good measure of local current densities. The results are presented in the following section.

\subsubsection{Analysis of the curlometer result}

The three components of the current have been computed in Solar Magnetic coordinates, and then transformed into local cylindrical co-ordinates, as shown in Fig. 9. As observed also in the particle data, sharp transitions appear in the current signatures, while the Cluster tetrahedron is entering the inner plasma sheet, as well as when it enters the ring current region. The plasma sheet is characterised by very nar-

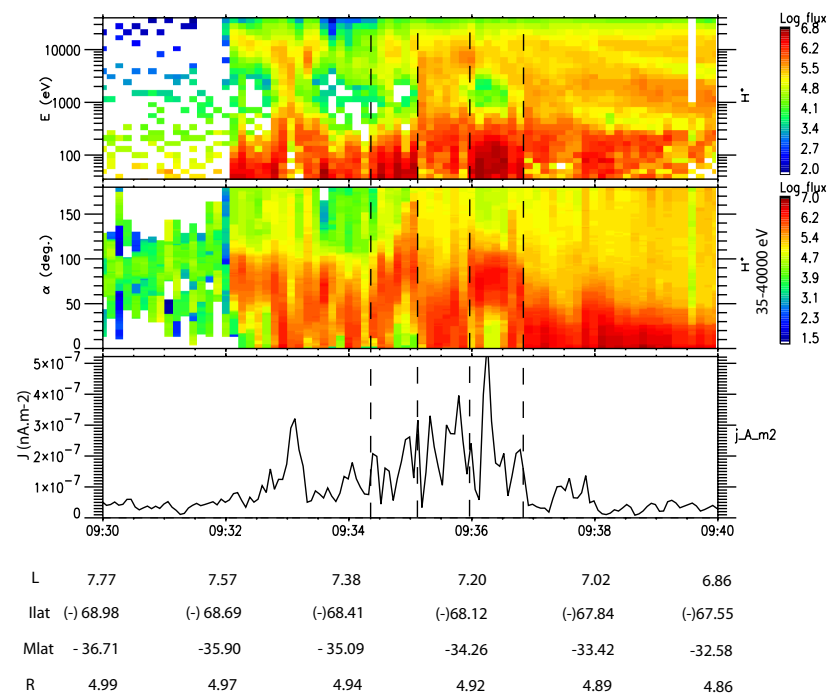

Fig. 10. Inner plasma sheet zoom for the 18 March 2002 event: $\mathrm{H}^{+}$ energy-time spectrogram in particle flux units (ions $/ \mathrm{cm}^{2} \mathrm{sr} \mathrm{s} \mathrm{keV}$ ), pitch angle distribution for the $35-40000 \mathrm{eV}$ energy range in particle flux units (ions $/ \mathrm{cm}^{2} \mathrm{sr} \mathrm{s} \mathrm{keV}$ ) and total current density in nA. $\mathrm{m}^{-2}$. L-shell, invariant latitudes, magnetic latitudes and geocentric distances are indicated below.

row "filamentations" of the current, very well correlated with the particle measurements (see Fig. 10). Each change in the ion population detected by the CIS instruments (i.e. alternation between perpendicular and field-aligned particles) is occurring simultaneously with an oscillation in the current density component. Note that the slight delay observed between these two types of measurements is due to the fact that the curlometer provides the current density averaged over the tetrahedron volume rather than the current density measured from the SC 4 location. These "filamentations" are representative of numerous traversals of superposed current layers. However, no conclusion concerning the current density values in this region can be made, since the oscillations observed in the $J$ profile characterise the nonlinearity of the current profile inside the tetrahedron rather than the absolute current of each layer crossed by the spacecraft. The "filamentations", which are of a scale smaller than the Cluster spacecraft separations, disappear as soon as the constellation enters the ring current region. In fact, the current density profile then becomes extremely smooth, almost linear all along the traversal until the entry into the northern inner plasma sheet (at 11:28 UT). In particular, the $J$ component along $\boldsymbol{e}_{\varphi}\left(J_{\varphi}\right)$ is very stable, with a standard deviation (between each plasma sheet crossing) of about $1.3 \mathrm{nA} . \mathrm{m}^{-2}$, whereas the mean $J_{\varphi}$ value is about -20 nA.m ${ }^{-2} \pm 20 \%$ (and the mean value of $\mathrm{J}_{\text {total }}$ is about $20.9 \mathrm{nA} \cdot \mathrm{m}^{-2} \pm 20 \%$ ). This negative value of $J_{\varphi}$ is in good agreement with the expected ring current flowing from dawn to dusk, i.e. oriented along the $-\boldsymbol{e}_{\varphi}$ direction (westward current). It appears thus that the FGM data show 

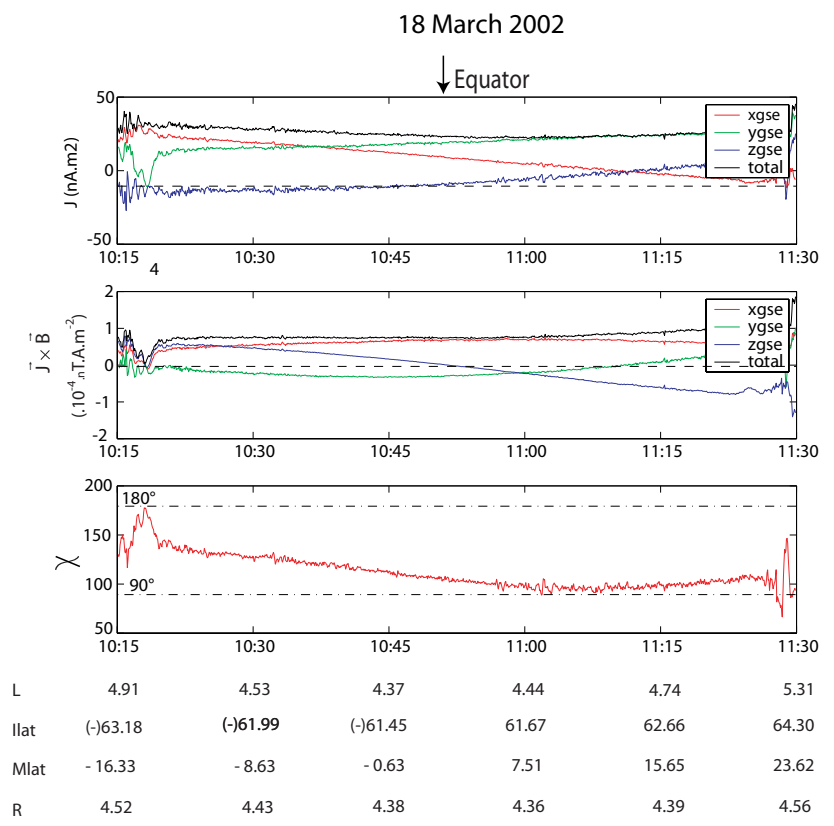

Fig. 11. Curlometer results for the 18 March 2002 event. From top to bottom: current density in the GSE coordinate system, pressure gradient (computed from $\boldsymbol{J} \times \boldsymbol{B}$ ), and $\chi$ angle between local current flow and magnetic field. L-shell, invariant latitudes, magnetic latitudes and geocentric distances are indicated at the bottom of the plot.

the existence of a residual westward ring current, even during very low activity periods $\left(D_{s t}>0\right)$. Furthermore, during weak activity, the very stable value of $J_{\varphi}$ shows that the ring current extends over the latitudinal range from Ilat $\sim 62.3^{\circ}$ $(\mathrm{SH})$ to $62.5^{\circ}(\mathrm{NH})$ for this MLT sector, i.e. between MLT $\sim 22$ and 00 (see also Ganushkina et al., 2002). Nevertheless, the contribution of $J_{\varphi}$ to the total current is decreasing at higher latitudes.

The other important current type expected in that region is field-aligned. To verify its presence, we plotted in Fig. 11 (third panel) the quantity $\chi=\cos ^{-1}(\boldsymbol{J} \bullet \boldsymbol{B} /\|\boldsymbol{J}\|\|\boldsymbol{B}\|)$, which gives the orientation of the current flow with respect to the magnetic field (averaged over the tetrahedron). Here again, the numerous alternations of this quantity in the inner plasma sheet, between $120^{\circ}$ and $180^{\circ}$, within a short time period (i.e. shorter than the maximum time separation between spacecraft) is mainly due to the fact that all spacecraft are not situated in the same current sheet. Moreover, considering that the maximum distance between spacecraft can reach $250 \mathrm{~km}$, and that their speed is about $5 \mathrm{~km} / \mathrm{s}$, we can't make any interpretation concerning structures (seen on the current components) for which their temporal extent is shorter than a minute. Narrow current signatures $(<1 \mathrm{~min})$ are necessarily inducing nonlinear magnetic field gradients inside the tetrahedron, since not all spacecraft are situated simultaneously in the same current sheet. Nevertheless, at about 10:17 UT, just before entering inside the ring current region (Ilat $=63^{\circ}, \mathrm{SH}$ ), a large peak ( $\sim 3$ min wide) is observed in the $\chi$ value, ap- proaching a value of $180^{\circ}$, characteristic of field-aligned currents flowing into the ionosphere. This field-aligned current location is coherent with the mapping established by Iijima and Potemra (1976), and is part of the region 2 current system. In the ring current region, the $\chi$ angle profile becomes then very smooth, its value decreasing from $\sim 130^{\circ}$ at high latitude to $\sim 90^{\circ}$ near the equator, which indicates that no field-aligned currents are present at those latitudes. This reveals the smooth transition in the ring current region, from a fully perpendicular current near the Equator to a more fieldaligned current at higher latitudes. Moreover, this shows the discontinuous transition of the current at the interface between the plasma sheet and the ring current.

Equation (2) states that electric currents in the ring current region must flow so as to balance the ion pressure gradient force: $\boldsymbol{J} \times \boldsymbol{B} \propto \operatorname{div}\left(\boldsymbol{P}_{\perp}\right)$. Panel 2 in Fig. 11 represents the $\boldsymbol{J} \times \boldsymbol{B}$ vector components. This quantity provides an estimate of the location of the maximum pressure. Error bars are not shown in that plot but they do not exceed $9 \%$ per component (considering a $20 \%$ error on $J_{z}$ and a $5 \%$ error on $J_{x}$ and $J_{y}$ ). It is worthy of note that the $\boldsymbol{J} \times \boldsymbol{B}$ amplitude (proportional to the pressure gradient) is almost constant over the entire ring current region, confirming the large latitudinal extent of ring current ion population. The inversion of the $\mathrm{z}$ component of $\boldsymbol{J} \times \boldsymbol{B}$ while crossing the equator justifies the assumption that the maximum ion pressure seems to be centred around this plane. The positive value of the $\mathrm{x}$ component all over the ring current region reveals that the maximum ion pressure is situated earthward with respect to the spacecraft, i.e. at $\mathrm{L}$-shell $<4.07$. This is consistent with the presence of a westward oriented current.

The lack of substantial particle fluxes measured by Cluster/CODIF (energy $<40 \mathrm{keV}$ ) during the perigee pass allow us to wonder how an azimuthal current of $\sim 20 \mathrm{nA} . \mathrm{m}^{-2}$ can flow, and which particles are likely to be the main current carriers. Williams et al. (1987) stated that $90 \%$ of the ring current carriers are situated within the $(15-250 \mathrm{keV})$ energy range. Above the CODIF energy range $(25 \mathrm{eV}-40 \mathrm{keV})$, the RAPID experiment on board Cluster provides ion data from $\sim 30 \mathrm{keV}$ to $1500 \mathrm{keV}$ (Wilken et al., 1997, 2001). Using these data, proton measurements reveal that an important flux increase (by about one order of magnitude in some energy bands) appears at higher energies, which peaks at $\sim 100$ to $400 \mathrm{keV}$ (third panel in Fig. 7). This would indicate that ring current carriers, for this event, have these higher energies, and that their energy distributions do not extent substantially below $40 \mathrm{keV}$. Please note that the current carried by these restricted energy range particles cannot be calculated from pressure gradient measurements, since the inter-experiment (CODIF and RAPID) and inter-spacecraft cross-calibration error bars do not allow for an accurate enough estimate of the pressure gradient. 


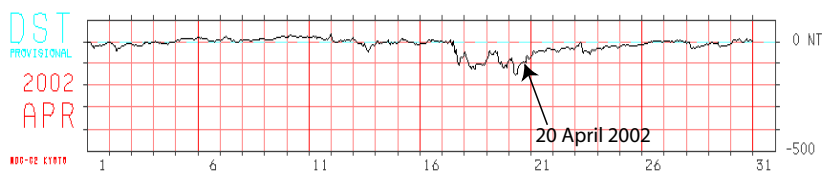

Fig. 12. $D_{s t}$ index values for April 2002. On the 20 April 2002 storm conditions are observed, while Cluster is passing through perigee. At 18:00 UT, the $D_{s t}$ index was about $-101 \mathrm{nT}$.

TANGO (SC 4) 20/Apr/2002

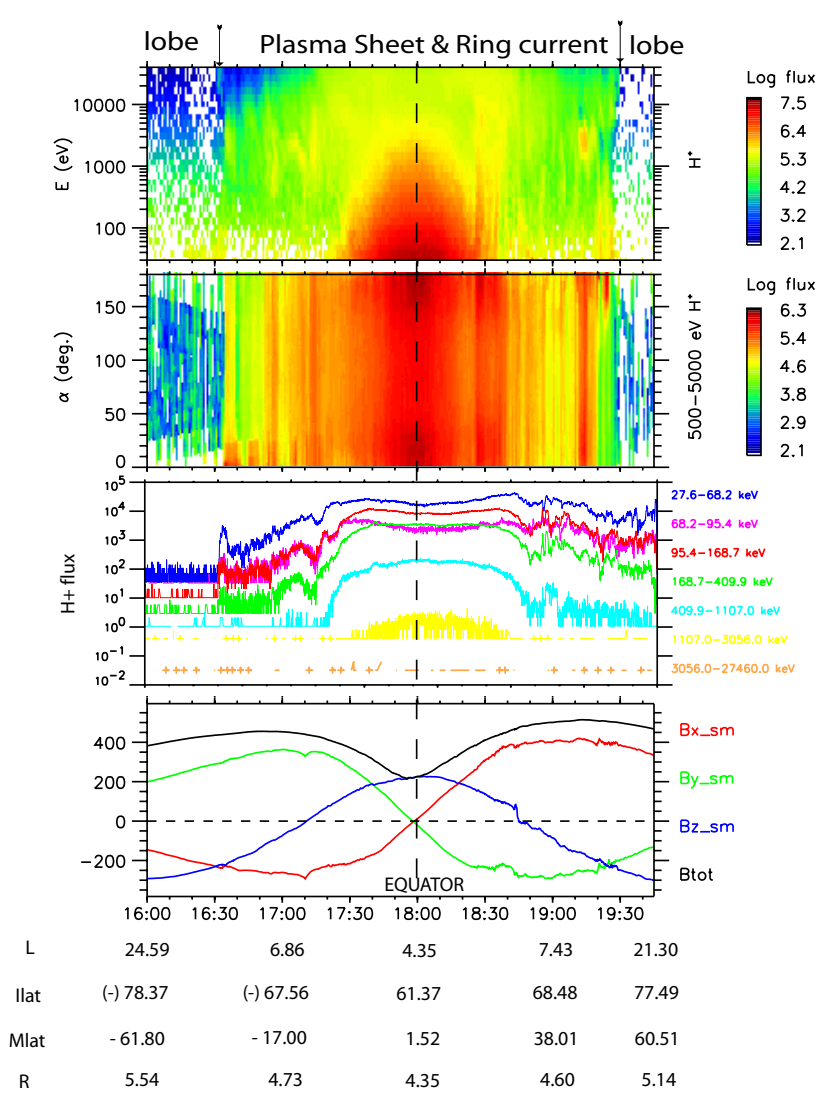

Fig. 13. Cluster SC4 data for the 20 April 2002 event: $\mathrm{H}^{+}$ energy-time spectrogram in particle flux units (ions $/ \mathrm{cm}^{2} \mathrm{srs} \mathrm{keV}$ ), the pitch-angle distribution (in particle flux units) for the 0.5 to $5 \mathrm{keV}$ energy range, the $\mathrm{H}+$ energy-time spectrogram from the RAPID instrument (27.6-3056.0 keV), and the magnetic field components in the SM coordinate system. L-shell, invariant latitudes, magnetic latitudes and geocentric distances are indicated below. Dashed line represents the equatorial crossing.

\subsection{Disturbed event: 20 April 2002}

\subsubsection{Context}

During this event the Cluster spacecraft were in the evening sector (MLT $\sim 21 \mathrm{~h}$ ). The $D_{s t}$ index for the interval (up to $-101 \mathrm{nT}$ at 18:00 UT) reveals a storm-time event, with a succession of storms (Fig. 12). The AE index (not shown) reached values up to $1000 \mathrm{nT}$ for this period. The $K_{p}$ value of 6 confirms this disturbance. Figure 13 represents the same type of plot as Fig. 7, but for the 20 April 2002 event. However, the pitch angle distribution was limited here to the 0.5 to $5 \mathrm{keV}$ energy range (CODIF and FGM data). Cluster SC4 entered from the southern lobe to the plasma sheet boundary layer at 16:30 UT, characterised by the presence of fieldaligned ions at low energies and the existence of very narrow structures observed for energies of $500 \mathrm{eV}$ to a few $\mathrm{keV}$. At lower latitudes (below Ilat $=62^{\circ}$ ), this population disappeared, and at about 17:14 UT a high energy population was encountered, showing the entrance in the ring current. The ring current population is characterised by a change in the pitch angle distribution, which became isotropic while approaching the equator.

Note that the profile is very different from the one recorded for the 18 March 2002 quiet time event, reflecting the intense magnetic activity level. This difference between quiet conditions and storm-time conditions also appears at higher energies, as revealed by the RAPID data. By comparing the 18 March (quiet, see Fig. 7, third panel) and 20 April 2002 (storm-time conditions, see Fig. 13, third panel) data, we note that RAPID records higher protons flux values during the storm-time event for particles with energies up to $95 \mathrm{keV}$. However, at higher energies, higher flux values are obtained during the quiet event.

Examining the FGM data for this event, they reveal perturbations on each of the components of the magnetic field, especially on the $B_{y}$ and $B_{z}$ components. By comparing the FGM data with the IGRF field, it appears that the measured magnetic field is much weaker than the IGRF one $\left(\left\|\mathrm{B}_{I G R F}\right\| /\left\|\mathrm{B}_{F G M}\right\| \sim 2\right.$ around the equator). We can thus conclude that here again the major part of the current contributing to the induced magnetic field is situated tailward of the spacecraft, with respect to the Earth.

\subsubsection{Analysis of the curlometer results}

The results of the current calculation in the ring current region appear in Fig. 14. Even if more disturbed than the event previously analysed, the transition from the plasma sheet to the ring current is still characterised by a change from a very oscillating current component profile until 17:43 UT (Ilat $\sim 61.3^{\circ}, \mathrm{SH}$ ) to a more regular one. Since the ring current population appears earlier on the particle data $(\sim 17: 14 \mathrm{UT})$, we can conclude that between 17:14 and 17:43, currents from the plasma sheet and the ring current are probably mixed, leading to nonlinear gradients inside the tetrahedron formed by the Cluster constellation and then to the presence of "filamentations" on the current profile. Once the filamentations disappear (after 17:43 UT), the current profile becomes smoother, even if it still shows a few small amplitude perturbations due to the high activity level, possibly related to multiple injections of particles. The dominant current component in the ring current region is, as expected, the $J_{\varphi}$ component, with a mean value of $J_{\varphi}=-24.6 \mathrm{nA} \cdot \mathrm{m}^{-2} \pm 20 \%$ (and the mean value of $\mathrm{J}_{\text {total }}$ is about $25.6 \mathrm{nA} \cdot \mathrm{m}^{-2} \pm 20 \%$ ). In spite of a much more intense magnetic activity level compared to 

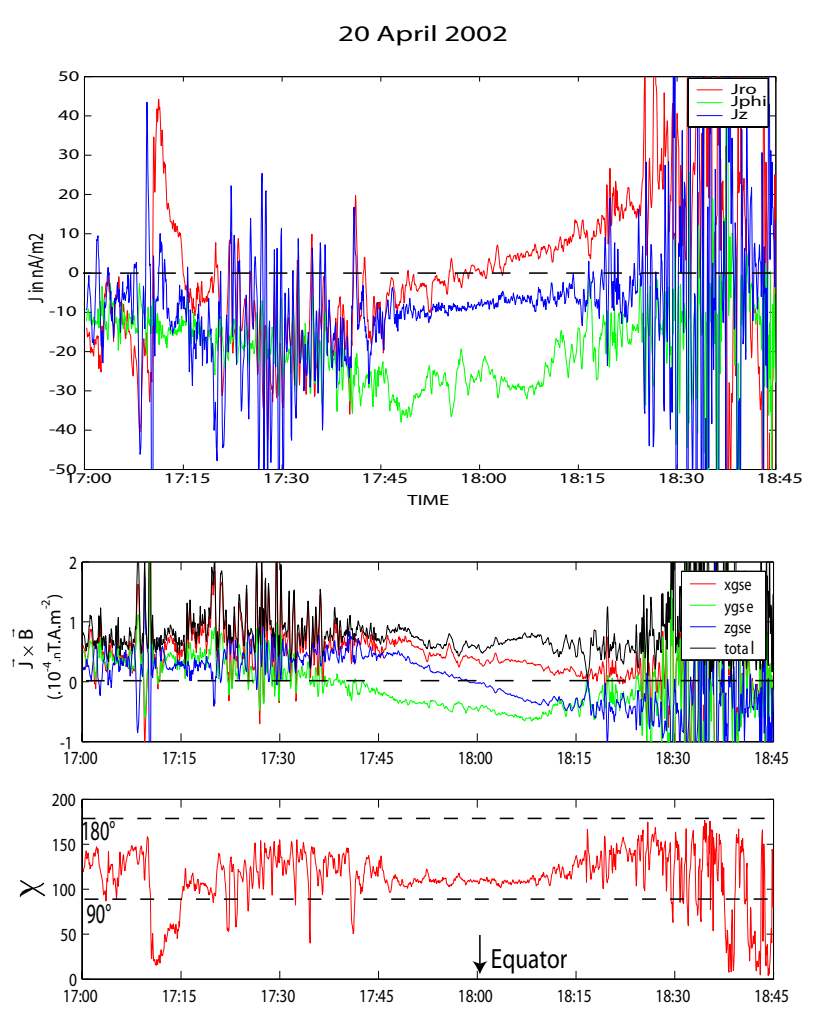

$\begin{array}{lcrcc}\text { L } & 6.86 & 4.87 & 4.35 & 5.00 \\ \text { Ilat } & (-) 67.56 & (-) 63.07 & 61.37 & 63.46 \\ \text { Mlat } & -34.07 & -17.00 & 1.52 & 20.30 \\ \text { R } & 4.73 & 4.47 & 4.35 & 4.40\end{array}$

Fig. 14. Curlometer results for the 20 April 2002 event. From top to bottom: current density in the local cylindrical coordinate system and in nA.m ${ }^{-2}$, pressure gradient (computed from $\boldsymbol{J} \times \boldsymbol{B}$ ), and $\chi$ angle between local current flow and magnetic field. L-shell, invariant latitudes, magnetic latitudes and geocentric distances are indicated at the bottom of the plot.

the previous event, the increase of the current density along the Cluster trajectory does not exceed $25 \%$. Nevertheless, considering that the curlometer technique allows for an estimation of the local current density rather than the total ring current intensity, this lack of strong correlation is not surprising. Since Cluster is situated at MLT 21, we expect a weaker current than closer to the midnight sector, where the bulk of the ring current is expected (Roelof et al., 2004). The $\boldsymbol{J} \times \boldsymbol{B}$ product, in Fig. 14, confirms this assumption since the negative $(\nabla P)_{y}$ value indicates that the main part of the current carriers is situated dawnward with respect to the spacecraft. Please note that for this event, and using the same assumptions as for the 18 March 2002 event, the error bars do not exceed $17 \%$ on each component. The $(\nabla P)_{x}$ is oriented earthward, which is consistent with the observed westward current. Concerning $(\nabla P)_{z}$, its inversion from positive values (in the Southern Hemisphere) to negative ones (in the Northern Hemisphere) lets us conclude that the ring current bulk is probably centred on the equatorial plane.
The current orientation (with respect to the magnetic field lines), given by the $\chi$ angle $\left(\chi=\cos ^{-1}(\boldsymbol{J} \bullet \boldsymbol{B} /\|\boldsymbol{J}\|\|\boldsymbol{B}\|)\right)$, is very stable all over the entire latitudinal extent of the ring current. Nevertheless, the current flow is not centred around $90^{\circ}$ but rather around $108^{\circ}$. If we look more carefully on the $J_{z}$ values for this event, we note a systematic southward orientation of the $J$ vector all along the traversal $\left(\sim 10 \mathrm{nA} . \mathrm{m}^{-2}\right)$. This stable negative value can only partially be explained by the $20 \%$ error made on the $J_{z}$ estimate (up to $8 \mathrm{nA} \cdot \mathrm{m}^{-2}$ ). If the current were fully perpendicular to $\boldsymbol{B}$ near the equator for this event, then a $0.8 \%$ gain uncertainty on $B_{z 1}$ would be required to explain such a negative value of $J_{z}$, using the curlometer. However, since the gain uncertainty is likely to always be below $0.02 \%$, we can conclude that a large part of this southward orientation is real and is due to the presence, near the equator, of a parallel component of the current density. This component could be the signature of an asymmetry between the ionospheric conductivities of the two hemispheres, which depend on the exposure to the sunlight. Since the exposure is different from one hemisphere to the other, and the closure of the ring current through the two hemispheres is a parallel circuit, this can drive field-aligned currents preferentially directed to one of the hemispheres (Hurtaud, 2004; Lu et al., 1994).

To estimate the ring current intensity $I_{R C}$ that would be required to produce the observed $D_{s t}$ (i.e. $D_{s t}=-101 \mathrm{nT}$ ), we use the reduced Biot-Savart formula:

$D_{s t}=\frac{1}{2} \cdot \mu_{0} \cdot \frac{I_{R C}}{R_{R C}-R_{E}}$

and the simplifying assumption that the bulk of the current is expected to be centred at a geocentric distance of $\sim 5 \mathrm{R}_{E}$. For this MLT sector, the total current needed to produce such a $D_{s t}$, and ignoring here the contribution of the quiet-time ring current, would then be $I_{R C} \sim 5.1 \times 10^{6} \mathrm{~A}$. Considering the observed extent of the ring current of $1.6 \mathrm{R}_{E}$ in the Zdirection and the measured current density of $\sim 30 \mathrm{nA} / \mathrm{m}^{2}$, this is equivalent to a radial extent of the bulk of the ring current of $\sim 2.6 \mathrm{R}_{E}$ (under the simplifying assumption of a uniform current density).

\section{Statistical study}

Periods for which the four Cluster spacecraft had a 100-km separation while crossing the cusp region were selected, corresponding to a separation at perigee short enough to give a realistic estimate of the current density. These separation distances were maintained for five months in 2002 (corresponding to approximately 60 perigee passes of Cluster), allowing for a survey of the ring current over a restricted portion of magnetic local times (see also Sect. 4.1). As a consequence, only the MLT sector from 17 to $01 \mathrm{~h}$ (dusk/postmidnight sector) has been covered. Nevertheless, FGM data weren't available for all perigee passes. Moreover, we had to remove events presenting eclipses (absence of valid data), or for which the transition from the plasma sheet into the ring 
Table 1. Cluster perigee passes (year 2002) used in the statistical study. For each pass is indicated the day, month, year, MLT sector crossed by Cluster at perigee, $D_{s t}$ index and the magnetic activity conditions.

\begin{tabular}{rccrrl}
\hline day & month & year & MLT & $D_{s t}$ & Conditions \\
\hline 6 & 2 & 2002 & 1.37 & -62 & storm peak \\
8 & 2 & 2002 & 1.7 & -20 & quiet \\
18 & 3 & 2002 & 22.96 & 13 & quiet \\
6 & 4 & 2002 & 21.72 & 10 & quiet \\
8 & 4 & 2002 & 21.8 & 12 & quiet \\
11 & 4 & 2002 & 21.5 & 25 & quiet \\
13 & 4 & 2002 & 21.33 & -40 & storm main phase \\
20 & 4 & 2002 & 21.0 & -91 & recovering \\
23 & 4 & 2002 & 20.9 & -23 & recovering \\
27 & 4 & 2002 & 20.7 & -4 & quiet \\
30 & 4 & 2002 & 20.31 & 8 & quiet \\
7 & 5 & 2002 & 19.7 & 4 & quiet \\
9 & 5 & 2002 & 19.8 & -2 & quiet \\
31 & 5 & 2002 & 18.33 & 6 & quiet \\
2 & 6 & 2002 & 18.0 & -13 & quiet \\
4 & 6 & 2002 & 18.3 & -10 & quiet \\
7 & 6 & 2002 & 17.6 & 7 & quiet \\
9 & 6 & 2002 & 17.66 & -10 & quiet \\
12 & 6 & 2002 & 17.7 & -12 & quiet \\
\hline
\end{tabular}

current did not clearly appear on the curlometer data (significant standard deviation in the data). This restricted our statistical study to 19 perigee passes in total (see Table 1). The current has been computed using the curlometer technique, and for each of these passes the tetrahedron parameters (elongation and planarity) have been carefully analysed, as well as their influence on the relative error made on $\boldsymbol{J}$, which has been evaluated using the study made by Robert et al. (1998b).

Figure $15 \mathrm{~b}$ shows the $\mathrm{X}_{S M}$ and $\mathrm{Y}_{S M}$ components of the current, for each selected event, as averaged in a $\pm 30^{\circ}$ invariant latitude interval around the equatorial plane, and projected down to the equator. The arrow lengths are proportional to the absolute value of the averaged equatorial current density. Figures 15a and c show the same kind of plots but for higher invariant latitudes, i.e. between 45 and $65^{\circ}$ above/below the equator in the Northern Hemisphere and in the Southern Hemisphere, respectively. Various magnetic activity levels have been included.

The statistics reproduce very well the general trend of the ring current, from the dusk sector to the post-midnight sector. All samples reveal a westward orientation of the current around the equator, consistent with the gradient and curvature drifts of the energetic protons and electrons. At higher latitudes (both in the Southern and in the Northern Hemispheres), we still observe the same feature, i.e. a westward orientation of the current. The azimuthal component of the westward current is still dominant at higher latitudes, confirming the large latitudinal extent of the ring current in that
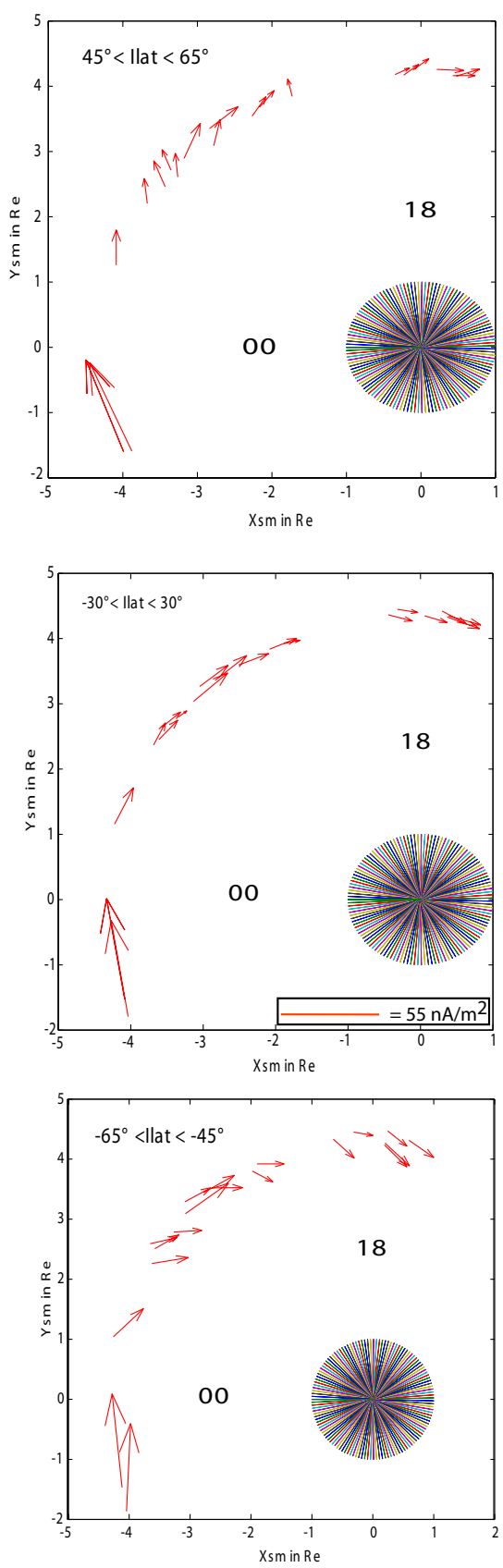

Fig. 15. Current density vectors for the February-June 2002 Cluster perigee passes, averaged over three invariant latitude intervals, and projected down to the equatorial plane: (a) from $45^{\circ}$ to $65^{\circ}$ (b) from $-30^{\circ}$ to $30^{\circ}$ (c) from $-45^{\circ}$ to $-65^{\circ}$

region (up to $65^{\circ}$ in invariant latitude), as previously seen for the 18 March and 20 April events. At these higher latitudes, particularly in the Southern Hemisphere, we also observe the increase of the radial component of the current, corresponding to the transition from a pure westward ring current to the Region 2 field-aligned currents.

The values of $J \rho$ and $J \varphi$ averaged over $30^{\circ}$ (invariant latitude) around the magnetic equator are plotted as a function 

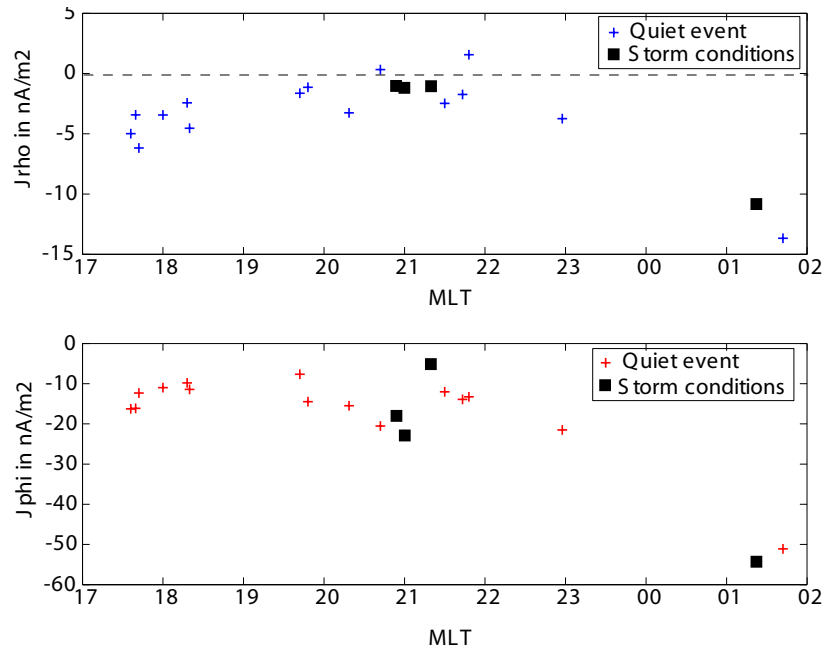

Fig. 16. Radial and azimuthal components of the current for the February-June 2002 Cluster perigee passes, as a function of the magnetic longitude, for the $-30^{\circ}$ to $30^{\circ}$ latitude interval, and projected down to the equatorial plane. Blue and red crosses represent the quiet events and black squares correspond to storm-time events.

of magnetic local time in Fig. 16. In this figure we also made the distinction between two different activity levels, based on the $D_{s t}$ index evolution, i.e. for quiet (events for which $D_{s t}>-20 \mathrm{nT}$ and presenting a smooth profile during the $24 \mathrm{~h}$ prior to the event, blue and red crosses) and storm phase conditions (black squares). In spite of the limited number of events, it appears that radial currents reach a minimum around MLT 21 and increase in absolute value away from this MLT sector. The maximum absolute values are observed in the post-midnight sector, at MLT 01 (see Fig. 16). This implies that azimuthal pressure gradients responsible for radial currents seem to be situated close to midnight (for the limited MLT sector considered here). However, the lack of orbital coverage over two-thirds of the magnetic local time and the limited number of events do not allow us to make conclusions about the azimuthal pressure global mapping.

Concerning the azimuthal currents, their density is higher by a factor of 2 to 5 , compared to the density of the radial component of the currents.

\section{Discussion}

In this paper the instantaneous current density measurements in the ring current were analysed for the first time using the curlometer technique. Two representative events have been presented, one corresponding to quiet magnetospheric conditions and the other corresponding to storm-time conditions. In the second part of this paper we presented results from a statistical study of over 19 perigee passes of the Cluster spacecraft.

Our results reproduce the westward orientation of the ring current, in the $\mathrm{L}=4$ to 5 range, and show its large latitudi- nal extent corresponding to a 2 to $4 \mathrm{R}_{E}$ thickness in the $Z$ direction, regardless of the geomagnetic activity level.

In spite of the limited number of storm-time events, we don't observe a substantial increase in the ring current density with increasing magnetospheric activity. For the two events studied in detail, the increase in the current density, from quiet conditions to the storm-time event, is of the order of $25 \%$. However, we should note that the curlometer technique does not supply the current intensity of the ring current bulk, but rather the current density along the trajectory of the center-of-mass of the spacecraft tetrahedron (orbital sampling effect). A way to position the Cluster spacecraft with respect to the ring current bulk is to use ENA image inversions to situate Cluster measurements into a global context (Vallat et al., 2004; Roelof et al., 2004). This was performed for the 20 April 2002 storm-time event and it showed that the Cluster spacecraft, at MLT $21 \mathrm{~h}$, crossed the ring current close to its westward edge, whereas the maximum of the ring current particles pressure, identified in the ENA image inversion, was close to midnight (Pontus C: Son Brandt, private communication).

Nevertheless, since the induced magnetic field generated by the ring current will tend to increase (or decrease, depending on the position of the measurement) the geomagnetic field amplitude, we used FGM data to calculate ( || $\mathrm{B}_{F G M} \|-$ $\left.\left\|\mathrm{B}_{I G R F}\right\|\right) /\left\|\mathrm{B}_{I G R F}\right\|$. This gives us an indication of the Cluster radial position with respect to the bulk of the ring current. The 20 April 2002 event analysis also indicates that the ring current bulk deduced from $\boldsymbol{J} \times \boldsymbol{B}$ (at least the maximum pressure responsible for the ring current) is situated eastward with respect to the spacecraft. This is consistent with the results of the ENA image inversions. Thus, the local $J$ measured with the curlometer technique does not fully reflect the intensification of the current. Note also that the lack of data available on the dayside doesn't permit us to make any conclusion about the development of the symmetric ring current over different storm phases. Furthermore, the study made by Le et al. (2004), which uses the curlometer technique with data issued from various spacecraft on different passes (and assuming stationarity), demonstrated the drift of the ring current bulk as a function of the $D_{s t} *$ index value. This study pointed out the evolution of the bulk position, from $\sim 6 \mathrm{R}_{E}$ far from the Earth (and centred in the midnight sector) during quiet periods to $\sim 5 \mathrm{R}_{E}$ (with a duskward drift) during storm-time periods. As a consequence, and since the Cluster perigee for the period considered is always situated at $\sim 4 R_{E}$ from the Earth, its position with respect to the bulk of the ring current does not necessarily reflect the total current intensification.

Jorgensen et al. (2004) used the same technique to map the magnetic field, but data have been sorted as a function of location and the $D_{s t}$ index value. Their mapping confirms that in the dusk and midnight sectors the maximum of the westward ring current is situated beyond the Cluster perigee distance (i.e. beyond $4 \mathrm{R}_{E}$ ), which is consistent with our results. From their study it appears that the Cluster orbit, at perigee $\left(\sim 4 \mathrm{R}_{E}\right)$, cuts the ring current in its westward (outer) 
component, but close to its inner edge, where the transition is observed to the eastward component of the ring current. There, as they show, the sensitivity of the ring current density to the $D_{s t}$ level is minimal, which is also consistent with our results. They also confirm the large latitudinal extent of the ring current at these distances.

However, in that region, (Jorgensen et al. (2004)) estimated the ring current densities from a few $\mathrm{nA} / \mathrm{m}^{2}$ during quiet events up to $\sim 10 \mathrm{nA} / \mathrm{m}^{2}$ during storm-time events, which is substantially lower than the results obtained here using Cluster-FGM data. Nevertheless, since the magnetic field data used for their study have been averaged in time and space, i.e. over one minute (while the FGM data used in our study have a time resolution of $\sim 4 \mathrm{~s}$ ) and for large spatial bins $\left(0.2 \mathrm{R}_{E}\right.$ by $0.2 \mathrm{R}_{E}$ by $3 \mathrm{~h}$ in MLT), the magnetic perturbation induced by the ring current has been smoothed, reducing the magnetic field gradients used in the current density calculations. This could explain, at least partially, an underestimation of the local ring current density, compared to the Cluster high-resolution simultaneous four-spacecraft data. Moreover, single spacecraft missions, on the contrary to multi-spacecraft ones, require the assumption of a stationary system over several orbit passes. This can induce an artefact in the current density estimate.

In our study we classified events as "quiet" and "storm" using the $D_{s t}$ index value. Nevertheless, it is worth noting that the quiet-time ring current is not represented by the $D_{s t}$ value, since it is a constant offset which is systematically subtracted from the ground stations B-field data (Mayaud, 1980; Rangarajan, 1989). Thus, a storm-time ring current will be characterised by an additional component, mainly carried by the so-called ring current particles, injected from the plasma sheet. This additional component appears on the $D_{s t}$ value.

As indicated earlier, by comparing the 18 March 2002 (quiet event, first panel of Fig. 11) and the 20 April events (disturbed event, first panel of Fig. 14), we observe an overall increase of $\sim 25 \%$ (from $\sim 21$ to $\sim 26 \mathrm{nA} / \mathrm{m}^{2}$ ) of the current density. This gives us an indication about the amount of current (carried by the injected particles) added to the quiet time one, in the evening sector.

To understand which are the main current carriers, it should also be noted that an important part of the quiet-time ring current may be carried by the radiation belt protons and electrons (MeV particles) (Boscher et al., 1998; Boscher and Bourdarie, 2001). This is revealed by the RAPID data for the 18 March 2002 event (Fig. 7), for which we observe an important flux of the high energy population (above $95 \mathrm{keV}$ ). Furthermore, for the 95-1100 keV energy range, the proton flux for this quiet-time event is significantly higher than that for the 20 April 2002 disturbed event (Fig. 13). Moreover, during each perigee pass, HIA (ion spectrometer without mass discrimination) almost always detects the outer radiation belts all along the equatorial pass in the form of background: HIA counting statistics are, in these cases, dominated by penetrating particles from the radiation belts. And even if no pressure gradient can be calculated directly, this confirms the assumption of a substantial flux gradient at very high energies which is capable of creating a current flow (data are not shown).

For the 18 March 2002 event we observe a field-aligned current flowing into the ionosphere at higher invariant latitudes $\left(\sim 62.5^{\circ}, \mathrm{SH}\right.$, see Fig. 11$)$. This current is certainly part of the Region 2 current system, as mapped by Iijima and Potemra (1976). For this MLT range, they observed the presence of current flowing into the ionosphere with a minimum invariant latitude of $\sim 63^{\circ}$, consistent with our results. The limited MLT range over which Cluster had suitably small tetrahedron separation did not, however, allow us to make conclusions about the field-aligned currents mapping in the other MLT sectors.

Our study also shows the very large latitudinal extent of the ring current, all over the evening and post-midnight sector. This has been predicted by Roelof $(1989,2004)$, who presented a zero-order global model of energetic ion distribution from energetic neutral atom images. Using Euler potentials, current densities were deduced from the ion pressure estimate. Roelof pointed out how radial pressure gradients are driving azimuthal currents, and how azimuthal pressure gradients (as a result of the midnight-noon asymmetry of the ring current) drive radial currents. The Cluster data reveal the large latitudinal extent of the ring current lines at invariant latitudes as high as Ilat $\sim 63^{\circ}$, for the MLT range considered. The Roelof model predicts the presence of such high latitude current lines, as the result of a non-zero component of $J$ parallel to $\boldsymbol{B}$, which leads lines into and out of the ionosphere, contributing to a "partial ring". Our results also reveal a systematic orientation of $(\mathrm{J} \rho)$ down to the ionosphere, whereas the Roelof model predicts outflowing currents in the post-midnight sector. These disagreements are partially due to certain assumptions used in the model, for example, quasisteady conditions, dipolar magnetic field, isotropic pitch angle distributions (whereas CIS data show structured pitch angle distributions, even near the equator), and a $\mathrm{J}_{/ /}$defined with an initial surface $\left(\mathrm{J}_{/ /}=0\right)$ at the magnetic equator. If we consider that the curlometer technique revealed the presence of a small, almost permanent southward orientation of $\boldsymbol{J}$ near the equator (for all MLT sectors considered, see upper panel of Fig. 14), this confirms that the assumption $J_{/ /}=0$ is no longer valid. This southward orientation indicates the presence of a field-aligned component of the current at very low latitudes. Note also that this model was developed for $\mathrm{L}=5$, whereas the Cluster spacecraft are passing through perigee at about $\mathrm{L} \sim 4$.

\section{Conclusion}

Cluster is the first mission to allow the estimate of the instantaneous local current density by using the curlometer technique. The spacecraft perigee location $\left(\sim 4 \mathrm{R}_{E}\right)$ allows an estimate of the ring current density at these distances.

Our analysis shows that the curlometer method is valid, in the ring current region, for inter-spacecraft separations at perigee up to $500 \mathrm{~km}$, and also depends on the tetrahedron 
parameters. Applying it over several perigee passes, we were able to produce very well the properties of the partial westward flowing ring current, from MLT $\sim 17$ to at least 01 (maximum MLT coverage available for Cluster and for the required tetrahedron geometry).

Our results reveal the systematic large latitudinal extent of the ring current (from $-65^{\circ}$ to $+65^{\circ}$ of invariant latitude, in average) and show the existence of a residual ring current around the midnight sector, even during very low activity periods. This permanent component of the ring current may be carried by the high energy particles of the radiation belts.

The relatively limited range of values for $J$ over all perigee passes is also related to the positioning of the spacecraft with respect to the bulk of the current.

The $\boldsymbol{J} \times \boldsymbol{B}$ product, proportional to the pressure gradient responsible for the current flow, appears to be systematically stable over the entire latitudinal extent of the ring current region.

The angle between $\boldsymbol{B}$ and $\boldsymbol{J}$ reveals the gradual evolution of the current density orientation from an almost fully perpendicular close to the equator to a more field-aligned orientation at higher latitudes, and the discontinuous transition at the interface between the plasma sheet and the ring current.

The current also presents, for the events studied, a systematic southward orientation in the ring current region, characterising the presence of a field-aligned component of the current near the equator. This orientation could be the consequence of the different ionospheric conductivities between the Northern and the Southern Hemisphere, leading to an asymmetry of the Region 2 field-aligned currents.

Acknowledgements. The $D_{s t}$ index was provided by the World data Center for Geomagnetism, Kyoto. The authors are indebted to P. W. Daly who provided us the RAPID data, and to P. C: Son Brandt for the ENA image inversions performed for the 20 April 2002 event.

Topical Editor T. Pulkkinen thanks P. Escoubet and another referee for their help in evaluating this paper.

\section{References}

Balogh, A., Dunlop, M. W., Cowley, S. W. H., Southwood, D. J., Thomlinson, J. G., Glassmeier, K.-H., Musmann, G., Luhr, H., Buchert, S., Acuna, M., Fairfield, D. H., Slavin, J. A., Riedler, W., Schwingenschuh, K., and Kivelson, M. G.: The Cluster magnetic field investigation, Space Sci. Rev., 79, 65-91, 1997.

Balogh, A., et al., The Cluster Magnetic Field Investigation: Overview of in-flight performance and initial results, Ann. Geophys.; 19, 1207-1217, 2001.

Boscher, D., Bourdarie, S., Friedel, R., Korth, A.: Long term dynamic radiation belt model low energy protons, Geophys. Res. Lett., 25, 22,4129-4132, 1998.

Boscher, D., S. Bourdarie, Modeling the radiation belts: what are the important physical processes to be taken into account in models?, Adv. Space. Res., 28, 12, 1739-1746, 2001.

Chanteur, G., Accuracy of field gradient estimations by Cluster: Explanation of its dependency upon elongation and planarity of the tetrahedron, in Proceeding of the Cluster-II Workshop Multiscale/ Multipoint Plasma Measurements, Imperial College, Lon- don, 1999, ESA SP-449, 265-268, European Space Agency, 2000.

Daglis , I. A. , Thorne, R. M., Baumjohann, W., and Orsini, S.: The terrestrial ring current: origin, formation and decay, Rev. Geophys., 37, 407-438, 1999.

Dandouras, I., Pierrard, V., Goldstein, J., Vallat, C., Parks, G. K.,Rème, H., Gouillart, C., Sevestre, F., McCarthy, M., Kistler, L. M., Klecker, B., Korth, A., Bavassano-Cattaneo, M. B., Escoubet, P., and Masson, A.: "Multipoint observations of ionic structures in the Plasmasphere by CLUSTER - CIS and comparisons with IMAGE-EUV observations and with Model Simulations", Yosemite 2004 AGU Monograph: Global Physics of the coupled inner magnetosphere, in press, 2005.

Dunlop, M. W., Southwood, D. J., Glassmeier, K. -H., and Neubauer, F. M: Analysis of multipoint magnetometer data, Adv. Space Res., 8, 9-10 , (9)273, 1988.

Dunlop, M. W. and Balogh, A.: On the analysis and interpretation of four spacecraft magnetic field measurements in terms of small scale plasma processes, in Spatio-temporal Analysis for Resolving Plasma Turbulence (START), Eur. Space Agency, WPP, ESA WPP-047, 223, 1993.

Dunlop, M. W., Balogh, A., Glassmeier, K. -H., and Robert, P.: Four-point Cluster application of magnetic field analysis tools: The Curlometer J. Geophys. Res., 107(A11), 1384-1398, 2002.

Escoubet, C. P., Fehringer, M., and Goldstein, M.: The Cluster mission, Ann. Geophys., 19, 1197-1200, 2001,

SRef-ID: 1432-0576/ag/2001-19-1197.

Ganushkina, N. Y., Pulkkinen, T. I., Kubyshkina, M. V., Singer, H. J., Russell, C. T.: Modeling the ring current magnetic field during storms, J. Geophys. Res. ,107(A7), 1092-1107, 2002.

Hurtaud, Y.: Modélisation de la dynamique couplée des plasmas magnétosphérique et ionosphérique, rapport de stage de DEA CESR, Toulouse, 2004.

Iijima, T. and Potemra, T. A.: The amplitude distribution of fieldaligned currents at northern high latitudes observed by Triad $\mathbf{J}$ Geophys. Res.,81(13), 2165-2174, 1976.

Jorgensen, A. M., Spence, H. E., Hughes, W. J., and Singer, H. J.: A statistical study of the global structure of the ring current, J. Geophys. Res., 109(A12204), 2004.

Le, G., Russell, C. T., and Takahashi, K.: Morphology of the ring current derived from magnetic field observations, Ann. Geophys., 22, 1267-1295, 2004,

SRef-ID: 1432-0576/ag/2004-22-1267.

Liemohn, M. W., Kozyra, J. U., Clauer, C. R., and Ridley, A. J.: Computational analysis of the near-Earth magnetospheric current system during two-phase decay storms, J. Geophys. Res., 106(A12), 29, 531-542, 2001.

Lu, G., Richmond, A. D., Emery, B. A.,,Reiff, P. H., de La Beaujardiere, O., Rich, F. J., Denig, W. F., Kroehl, H. W., Lyons, L. R., Ruohoiemi, J. M.: Interhemispheric asymmetry of the high-latitude ionospheric convection pattern, J. Geophys. Res., 99(A4), 6491-6510, 1994.

Lui, A. T. Y.: Characteristics of the cross-tail current in the earth's magnetotail, in "Magnetospheric currents", Geophys. Monogr Ser., 28, (Ed.) Potemra, T. A., 158, AGU, Washington D.C., 1984.

Lui, A. T. Y., McEntire, R. W., and Krimigis, S. M.: Evolution of the Ring Current during two geomagnetic storms, J. Geophys. Res., 92(A7), 7459-7470,1987.

Mayaud, P. N.: Derivation, Meaning, and Use of Geomagnetic Indices, Geophysical Monograph 22, AGU, Washington D.C., 1980 . 
Milillo, A., Orsini, S., Delcourt, D. C., Mura, A., Massetti, S., and DeAngelis, E.: Empirical model of proton fluxes in the equatorial inner magnetosphere: 2. Properties and applications, J. Geophys. Res., 108(A5),1165-1178, 2003.

Mitchell, D. G., Williams, D. J., Huang, C. Y., Frank, L. A., and Russell, C. T.: Current carriers in the near-Earth cross-tail current sheet during substorm growth phase, Geophys. Res. Lett., 17, 583-586, 1990 .

Rangarajan, G. K.: Indices of geomagnetic activity, in Geomagnetism, 323, (Ed.) J. A. Jacobs, Academic Press, London, 1989.

Rème, H., Aoustin, C., Bosqued, J., Dandouras, I., Lavraud, B., Sauvaud, J. A., Barthe, A., Bouyssou, J., Camus, Th., CoeurJoly, O., et al.: First multispacecraft ion measurements in and near the Earth's magnetosphere with the identical Cluster ion spectrometry (CIS) experiment, Ann. Geophys., 19, 1303-1354, 2001,

\section{SRef-ID: 1432-0576/ag/2001-19-1303.}

Robert, P. and Roux, A.: Dependence of the shape of the tetrahedron on the accuracy of the estimate of the current density, in Spatiotemporal Analysis for Resolving Plasma Turbulence (START), Eur. Space Agency WPP, ESA WPP-047, 289-293, 1993.

Robert, P., Roux , A., Harvey, C. C., Dunlop, M., Daly, P. W., and Glassmeier, K.-H.: Tetrahedron Geometric Factors, Analysis Methods for Multi-Spacecraft data, ISSI Sci. Rep. SR-001, 323-348, 1998a.

Robert, P., Dunlop, M. W., Roux, A., and Chanteur, G.: Accuracy of current density determination, in Analysis Methods for MultiSpacecraft data, ISSI Sci. Rep. SR-001, 395-418, 1998b.

Roelof, E. C.: Remote sensing of the ring current using energetic neutral atoms, Adv. Space Res., 9(12), 12 195-12 203, 1989.

Roelof, E. C., Son Brandt, P. C, and Mitchell, D. G.: Derivation of currents and diamagnetic effects from global plasma pressure distributions obtained by IMAGE/HENA, Adv. Space Res., 33, 747-751, 2004.
Singer, S. F.: A new model of magnetic storms and aurorae, Eos Trans. AGU, 38, 175-190, 1957.

Vallat, C., Dandouras, I., Son. Brandt, P. C, DeMajistre, R., Mitchell, D. G., Roelof, E. C., Rème, H., Sauvaud, J.-A., Kistler, L., Mouikis, C., Dunlop, M., and Balogh, A.: First comparisons of local ion measurements in the inner magnetosphere with energetic neutral atom magnetospheric image inversions: Cluster-CIS and IMAGE-HENA observations, J. Geophys. Res., 109(A04213), doi:10.1029/2003JA010224, 2004.

Wilken, B., Axford, W. I., Daglis, I., Daly, P., Güttler, W., Ip, W. H., Korth, A., Kremser, G., Livi, S., Vasyliunas, V. M., Woch, J., Baker, D., Belian, R. D., Blake, J. B., Fennell, J. E., Lyons, L. R., Borg, H., Fritz, T. A., Gliem, F., Rathje, R., Grande, M., Hall, D., Kecsueméty, K., McKenna-Lawlor, S., Mursula, K., Tanskanen, P., Pu, Z., Sandahl, I., Sarris, E. T., Scholer, M., Schulz, M., Sørass, F., and Ullaland, S.: RAPID—The imaging energetic particle spectrometer on Cluster, Space Sci. Rev., 79, 399-473, 1997.

Wilken, B., Daly, P. W., Mall, U., Aarsnes, K., Baker, D. N., Belian, R. D., Blake, J. B., Borg, H., Büchner, J., Carter, M., Fennell, J. F., Friedel, R., Fritz, T. A., Gliem, F., Grande, M., Kecskemety, K., Kettmann, G., Korth, A., Livi, S., McKenna-Lawlor, S., Mursula, K., Nikutowski, B., Perry, C. H., Pu, Z. Y., Roeder, J., Reeves, G. D., Sarris, E. T., Sandahl, I., Søraas, F., Woch, J., and Zong, Q.-G.: First results from the RAPID imaging energetic particle spectrometer on board Cluster, Ann. Geophys., 19, 1355-1366, 2001,

SRef-ID: 1432-0576/ag/2001-19-1355.

Williams, D. J.: Ring current and radiation belts, U.S. Natl. Rep. Int. Union Geod. Geophys. 1983-1986, Rev. Geophys., 25, 570578, 1987. 